\title{
Time-independent gravitational fields in the BGK scheme for hydrodynamics
}

\author{
A. Slyz ${ }^{1,2}$ and K.H. Prendergast ${ }^{1}$ \\ 1 Columbia University, 10027 New York, U.S.A. \\ 2 Max-Planck Institut für Astronomie, Königstuhl 17, 69117 Heidelberg, Germany
}

Received March 30; accepted April 21, 1999

\begin{abstract}
We incorporate a time-independent gravitational field into the BGK scheme for numerical hydrodynamics. In the BGK scheme the gas evolves via an approximation to the collisional Boltzmann equation, namely the Bhatnagar-Gross-Krook (BGK) equation. Time-dependent hydrodynamical fluxes are computed from local solutions of the BGK equation. By accounting for particle collisions, the fundamental mechanism for generating dissipation in gas flow, a scheme based on the BGK equation gives solutions to the Navier-Stokes equations: the fluxes carry both advective and dissipative terms. We perform numerical experiments in both 1D Cartesian geometries and axisymmetric cylindrical coordinates.
\end{abstract}

Key words: hydrodynamics — methods: numerical — shock waves - gravitation

\section{Introduction}

The BGK code distinguishes itself from other hydrocodes in that it has recourse to the physics which generates dissipation, namely the physics of particle collisions. Developed by Prendergast \& Xu (1993), the hydrodynamical scheme invokes a microscopic description of gas flow and it is therefore based on considerations of gas kinetic theory. Recall that kinetic-based hydrocodes rely on the fact that the state of a gas can be described by giving the distribution function of particle velocities $f(\mathbf{x}, \mathbf{u}, t)$ at a point in the phase space of a single particle. The codes take advantage of the fact that the quantities of hydrodynamical interest (namely the mass, momentum and energy densities in the gas) are low-order velocity moments of $f$. $f$ obeys the Boltzmann-equation, which we write as $\frac{D f}{D t}=\frac{\delta f}{\delta t}^{\text {coll }}$, where $\frac{D}{D t}$ is a time rate of change along the trajectory of a single particle moving freely in phase space (under the action of smoothly varying forces, if these are present) and $\frac{\delta f}{\delta t}^{\text {coll }}$ is the rate of change of $f$ due to collisions. The classical two-body collision integral is non-linear, and non-local in velocities (Cercignani 1988). BGK (from the names of Bhatnagar-Gross-Krook) replaces this integral with the term $\frac{(g-f)}{\tau}$, where $g$ is the Maxwell-Boltzmann distribution function having the same mass, momentum and energy densities as $f$, and $\tau$ is a relaxation time, which can (ideally) be as small as the mean time between collisions of a particle in the gas (Bhatnagar et al. 1954). It is important to notice that $f$ and $g$ have the same mass, momentum and energy densities, but they do not give rise to the same fluxes of these quantities. Fluxes are determined by different moments of the distribution functions. BGK differs from all other kinetic-based codes in several respects: the collisions are active throughout the duration of a time step, and are not imposed as a distinct "equilibration" process at the end of a timestep. Also, we do not guess the form of the distribution function but solve the BGK equation to find it. The BGK equation is linear in $f$, which makes it easy to solve if $g$ is known. However $g$ is not known; the "compatability" conditions that $f$ and $g$ have the same mass, momentum and energy densities would determine $g$ if $f$ were known. Therefore the BGK formulation is also nonlinear and non-local in velocity space, as is the Boltzmann equation. It will be shown that this situation leads to a set of non-linear integral equations for the parameters of $g$. It might seem that we have made no progress by replacing the Boltzmann equation by the BGK plus compatability conditions; but this is not true, because it will be shown that we need solve for the parameters of $g$ only in the neighborhood of a boundary between computational cells, and for a short time (given by the usual CFL (Courant-FriedrichsLewy) condition). Knowledge of the parameters of $g$ is

Send offprint requests to: slyz@mpia-hd.mpg.de 
equivalent to knowledge of the mass, energy and momentum densities.

The connection between the BGK equation's microscopic description of gas flow and a macroscopic description has been shown by Cercignani (1988) for the case of a perfect monatomic gas and by Xu (1993) for polyatomic gases. Velocity moments of the BGK equation give the Euler equations for negligible particle collision time, $\tau$, the Navier-Stokes equation for small yet non-zero $\tau$ and a description of rarefied gas dynamics for large $\tau$. In the Navier-Stokes regime, these derivations also furnish expressions for the shear and bulk viscosity coefficients and the heat conductivity coefficient.

In principle, if the local value of the collision time can be measured as a function of time for a gas, then the BGK scheme may be used to evolve the gas with true physical dissipation parameters. In practice, the resolution of the BGK scheme is limited by the grid resolution. If the grid is not fine enough to resolve a discontinuity, then artificial dissipation must be added to broaden the discontinuity so that it is at least one grid cell thick. Because viscosity and heat conductivity are proportional to $\tau$, the BGK scheme broadens shocks by enlarging $\tau$ at the location of the discontinuities. The expression for the collision time in the BGK scheme therefore contains two terms. One term is the real physical mean collision time and it is chosen according to the desired Reynolds number of the problem. The second term is chosen in such a way that shocks in the flow span at least one grid cell. The latter term tunes the amount of artificial dissipation in the scheme. The notable difference between how the BGK scheme inputs artificial dissipation and how other schemes input artificial dissipation is that the BGK scheme puts it in exactly as if it were real dissipation corresponding to the numerically necessary value for $\tau$.

We emphasize that modelling the real dissipation in an astrophysical object such as that resulting from "turbulent" viscosity, is still outside the reach of any existing hydrocode. For one thing, values for viscosities in astrophysical objects are highly speculative. Secondly, to solve Navier-Stokes problems with a particular value of the viscosity for an astrophysical object requires grid resolutions which are beyond what is currently achievable. For laboratory scale phenomena and for small enough Reynolds number, the BGK code is successful at modelling the dissipation produced by particle collisions in a fluid. Tests of the Kolmogorov and the laminar boundary layer problem show real dissipative effects (Xu \& Prendergast 1994).

Thus far the BGK code has been extensively tested without the inclusion of gravity. Several papers document the tests which verify its accuracy and robustness as both an Euler and Navier-Stokes solver in 1D and 2D Cartesian geometries and for two-dimensional adaptive unstructured grids. The list of one-dimensional Euler tests which have been performed with the BGK scheme includes: the Roe, Sod, Lax-Harten, Woodward-Colella, and Sjögreen tests for subsonic and supersonic expansion (Prendergast \& Xu 1993; Xu 1993; Xu et al. 1995). The results from these onedimensional test cases are that the BGK scheme produces shock fronts which are typically one to two cells wide, and contact discontinuities which are slightly broader. This is competitive with high resolution codes which do not employ regridding in the neighborhood of a shock front. The BGK scheme also exhibits negligible under and over shooting even at strong shock fronts. The other notable features in the tests with rarefaction waves are the sharp corners at the junctions between the rarefaction waves and the undisturbed, uniform regions.

Many codes have trouble treating a low density region with a flow gradient. The BGK scheme successfully handles low density regions because it satisfies both an entropy condition and a positivity condition (Xu et al. 1996). In the BGK scheme collisions relax the gas toward local thermodynamic equilibrium states and this relaxation process is accompanied by an increase in entropy. Godunovtype schemes on the other hand typically demand an entropy fix when they encounter strong rarefaction waves (cf. the Sjögreen test) otherwise they produce unphysical rarefaction shocks. Usually the fix is an addition of artificial viscosity. Because the BGK scheme naturally satisfies the entropy condition, it simply cannot generate these unphysical phenomena. In satisfying the positivity condition, the BGK scheme avoids producing states with negative density or internal energy. The Roe test is conducive to the creation of these non-physical states but the BGK scheme does not encounter them (Prendergast \& Xu 1993). The same cannot be said of the performance of conservative finite difference schemes and codes employing Riemann solvers (e.g. Roe's approximate Riemann solver).

The list of two-dimensional Euler test cases which have been performed with the BGK scheme includes: (a) uniform Mach 3 flow in a tunnel with a forward facing step (the Emery test). The BGK code is not modified in any way near the step to treat the flow past it and its corner, which is a singular point. With the BGK scheme expansion shocks never emerge from the corner (Prendergast \& Xu 1993; Xu 1993; Xu et al. 1995), (b) double Mach reflection in supersonic flow over a wedge, (c) the diffraction of a strong shock $($ Mach $=5.09)$ around a corner. Test cases (b) and (c) are further examples of the BGK scheme succeeding at simulating flow without having to summon any detection algorithms or entropy fixes. According to $\mathrm{Xu}$ et al. (1995), the original Godunov scheme, the Roe scheme without the entropy fix, and the Osher scheme could produce a rarefaction shock at the corner in test case (c); (d) flow around an impulsively started cylinder. When applied to this problem, many schemes either fail or have severe problems in maintaining positive pressure and density in the near vacuum low pressure and low density region created behind the cylinder. The BGK scheme seems to be able to preserve positivity without ad hoc 
fixes, and to reach a steady state solution for the problem (Xu et al. 1996).

To test its performance as a Navier-Stokes solver and to see if it has real viscosity effects, the BGK scheme has been applied to the laminar boundary layer problem and the Kolmogorov problem ( $\mathrm{Xu}$ 1993). The laminar boundary layer problem models the flow of gas above a flat plate. Even on coarse grids (e.g. (32 X 16), (16 X 8)), the BGK scheme impressively recovers the Blasius profile. In the Kolmogorov problem, a one-dimensional sinusoidal velocity field is imposed in a uniform density and isothermal fluid. The BGK code fulfills the Navier-Stokes prediction which is that the shape of the fluid's velocity profile remains unchanged while the amplitude of the velocity decreases in such a way that the fluid's kinetic energy decays exponentially. The agreement between the theoretical viscosity coefficient and the numerical viscosity coefficient (deduced from the measured decay rate) is excellent.

To improve the resolution of physical discontinuities occurring in complicated flows, a version of the BGK scheme on a two-dimensional adaptive unstructured grid has now been developed (Kim \& Jameson 1998).

Most astronomical applications of a hydrocode require a consideration of gravity. To this end we have been developing the BGK scheme. Recently the BGK scheme has been used for a cosmological simulation (Xu 1997) but this is prior to results showing the long-term stability of the BGK scheme with gravity and the BGK scheme's convergence to the equilibrium state with gravity. In this paper we give such results. In addition to the incorporation of gravity, we have modified the geometry of the Eulerian grid to axisymmetric cylindrical coordinates. Changing the geometry of the grid has effects similar to the effects of adding gravity to the BGK scheme. It gives rise to source terms in the hydrodynamic equations. For the purpose of simplicity, in this paper we describe modifications to the BGK method when a time-independent gravitational potential is incorporated into a BGK scheme in Cartesian coordinates (Sect. 2). We present results however for both gas flow on a one-dimensional Cartesian grid and on an axisymmetric cylindrical grid (Sect. 3).

\section{A BGk flow solver with gravity}

\subsection{Hydrodynamic equations from the BGK equation with gravity}

In Cartesian coordinates, we specify the position and velocity of a particle with coordinates $(\mathbf{x}, \mathbf{u})$. To be clear, $\mathbf{x}$ here is a 3 -vector $(x, y, z)$ giving the position of a particle in configuration space and $\mathbf{u}$ is a 3 -vector $(u, v, w)$ giving the velocity of a particle in this space. In this coordinate system and in the presence of a smooth gravitational field with gravitational potential, $\Phi(\mathbf{x})$, the BGK equation is:

$\frac{\partial f}{\partial t}+\mathbf{u} \cdot \frac{\partial f}{\partial \mathbf{x}}-\frac{\partial \Phi}{\partial \mathbf{x}} \cdot \frac{\partial f}{\partial \mathbf{u}}=\frac{g-f}{\tau}$.
(For reference, this is equivalent to Eq. (2.15) in Shu (1992) where $\frac{\delta f}{\delta t}^{\text {coll }}$ is the BGK collision term $\frac{g-f}{\tau}$.) Recall that in the BGK collision term $g$ is the equilibrium distribution towards which the true velocity distribution function $f$ relaxes on a collision timescale, $\tau$. We define the units of $f$ and $g$ to be the mass in a volume element of phase space. The particle collision time $\tau$ depends on macroscopic quantities such as temperature and density, and it is therefore a function of position $\mathbf{x}$ and time $t$. Both $f$ and $g$ are functions of space $\mathbf{x}$, time $t$, and particle velocities $\mathbf{u}$ and $\xi$ where $\xi=\left(\xi_{1}, \xi_{2}, \ldots \xi_{K}\right)$ is a $K$-dimensional vector of velocities associated with the internal degrees of freedom of a particle. With this parameterization of the internal velocities, the energy associated with internal degrees of freedom is written as $\frac{1}{2} m \xi^{2}$ where $\xi^{2}=\xi_{1}{ }^{2}+\xi_{2}{ }^{2}+\ldots+\xi_{K}{ }^{2}$. Included in these internal degrees of freedom is the energy not explicitly accounted for in versions of the code with physical spatial dimension, $D$, less than three. For example a particle in a diatomic gas $(\gamma=7 / 5)$ moving in 3 spatial dimensions has 5 degrees of freedom. (The connection between the number of degrees of freedom, $n$, and the ratio of specific heats, $\gamma$, is $\gamma=(n+2) / n$.) Because a one dimensional hydrocode $(D=1)$ treats only one of these degrees of freedom explicitly, the behavior of the diatomic gas is reproduced by treating the four remaining degrees of freedom as internal energies. We represent those energies in the K-dimensional vector $\xi$ where $K=n-D$. In terms of $\gamma, K=\frac{2}{(\gamma-1)}-D$.

Equations governing the time evolution of the mass density, momentum density and kinetic plus internal energy density are found by multiplying (1) in turn by $1, \mathbf{u}$, and $\frac{1}{2}\left(\mathbf{u}^{2}+\xi^{2}\right)$ and integrating over a volume in velocity space, $\mathrm{d} \Xi=\mathrm{d} \mathbf{u} \xi^{(K-1)} \mathrm{d} \xi$. The volume element in velocity space has the above form in the $\xi$ variable because we need only the total internal energy of a particle and not the energy associated with any particular internal degree of freedom. Hence an integration has been performed over angles in $\xi$. An essential point about the derivation of the hydrodynamic equations from velocity moments of the BGK equation is that moments of the BGK collision term, $\frac{(g-f)}{\tau}$, vanish. This is enforced by the fundamental hypothesis of the BGK model: in the relaxation process collisions tend to reshuffle the particles defining the true distribution function, $f(\mathbf{x}, \mathbf{u}, t)$ into a local equilibrium distribution, $g(\mathbf{x}, \mathbf{u}, t)$ having the same mass, momentum and energy densities as $f(\mathbf{x}, \mathbf{u}, t)$. We call this requirement for the equivalence of $f$ and $g$ 's moments, the "compatability condition".

For detailed results of taking velocity moments of (1), we refer once again to Shu (1992) (p. 20-23). We give the moment equations here in compact form:

$\frac{\partial}{\partial t}\langle\zeta\rangle+\frac{\partial}{\partial x_{k}}\left\langle u_{k} \zeta\right\rangle+\frac{\partial \Phi}{\partial x_{k}}\left\langle\frac{\partial \zeta}{\partial u_{k}}\right\rangle=0$ 
where we have defined the following bracket notation:

$\langle\ldots\rangle=\int(\ldots) f \mathrm{~d} \Xi$

and $\zeta=1, u_{i}, \frac{1}{2}\left(\mathbf{u}^{2}+\xi^{2}\right)$. In Eq. (2) we see that mass, momentum and energy densities are updated by terms which are the divergence of a quantity (i.e. this quantity is commonly referred to as a flux) and when gravity (or a curved coordinate system) is involved, also by terms which cannot generally be written as the divergence of a quantity and hence cannot update the contents of a cell by a flux through the cell's surface. The latter terms are called source terms. Without gravity and in Cartesian coordinates, a BGK scheme for hydrodynamics evolves all the hydrodynamic quantities exclusively by flux terms. We emphasize that there is no dissipation source term in Eq. (2). Dissipative terms are carried by the fluxes. The BGK scheme is unique in this respect. In Cartesian coordinates it allows a fluid to evolve concurrently through advective and dissipative processes without decoupling them into two separate operations.

With gravity, source term computation is unavoidable. The gravitational source term in the momentum equation cannot be manipulated into the form of the divergence of some quantity, so it persists as a source term. The gravitational source term in the energy equation on the other hand may be reformulated to give an energy equation without a source term if the gravitational field is independent of time. In this paper we consider this case. Without a gravitational source term, the energy equation for the case of a time-independent gravitational field is a conservative equation for the total kinetic, internal and gravitational energies. In one dimension it is:

$\frac{\partial}{\partial t}\left(\mathcal{E}_{\text {kin }}+\mathcal{E}_{\text {int }}+\rho \Phi\right)++\frac{\partial}{\partial x}\left(F^{\mathcal{E}_{\text {kin }}+\mathcal{E}_{\text {int }}}+\Phi F^{\rho}\right)=0$.

We define $F^{\mathcal{E}_{\text {kin }}+\mathcal{E}_{\text {int }}}$ here as the flux of the kinetic plus internal energy, and $F^{\rho}$ as the mass flux. In $F^{\mathcal{E}_{\text {kin }}+\mathcal{E}_{\text {int }}}$ and $F^{\rho}$ we are only considering the $x$-component of the flux, i.e. the flux through a wall perpendicular to the $x$ direction.

In the end, the BGK scheme follows the time evolution of the integrated values of mass, momentum and energy densities within cells. For example, for one dimensional flow in Cartesian coordinates the BGK scheme tracks the contents of a volume element with boundaries $x_{1}$ and $x_{2}$. If the values of mass, momentum and energy densities (denoted below by $q$ ) are given within this cell at time $t_{1}$ then at time $t_{2}$ the new values of the mass, momentum and energies are updated by fluxes $(\mathcal{F})$ (Sect. 2.2) through the cell's boundaries and in some cases by source terms $(S)$ (Sect. 2.7).

$$
\begin{aligned}
& \int_{x_{1}}^{x_{2}} q\left(x, t_{2}\right) \mathrm{d} x=\int_{x_{1}}^{x_{2}} q\left(x, t_{1}\right) \mathrm{d} x+\int_{t_{1}}^{t_{2}} \mathcal{F}\left(x_{1}, t\right) \cdot \hat{\mathbf{x}} \mathrm{d} t \\
& -\int_{t_{1}}^{t_{2}} \mathcal{F}\left(x_{2}, t\right) \cdot \hat{\mathbf{x}} \mathrm{d} t+\int_{t_{1}}^{t_{2}} \int_{x_{1}}^{x_{2}} S(x, t) \mathrm{d} x \mathrm{~d} t .
\end{aligned}
$$

\subsection{Hydrodynamical fluxes with gravity}

Fluxes in the BGK scheme arise from velocity moments of a particle distribution function $f$ which is a solution to the ordinary differential equation $\frac{D f}{D t}+\frac{f}{\tau}=\frac{g}{\tau}$ with initial conditions $f=f_{0}$ at $t=t_{0}=0$. This equation holds along each trajectory. The solution is composed of two terms: an integral over the past history of $g$ and a term representing relaxation from an initial state, $f_{0}$.

$$
\begin{aligned}
f\left(\mathbf{x}_{\mathrm{f}}, \mathbf{u}, t\right) & =\frac{1}{\tau} \int_{0}^{t} g\left(\mathbf{x}^{\prime}, \mathbf{u}^{\prime}, t^{\prime}\right) \mathrm{e}^{-\left(t-t^{\prime}\right) / \tau} \mathrm{d} t^{\prime} \\
& +\mathrm{e}^{-t / \tau} f_{0}\left(\mathbf{x}_{\mathrm{f}}-\mathbf{u} t, \mathbf{u}, t_{0}\right) .
\end{aligned}
$$

Here $\mathbf{x}^{\prime}$ and $\mathbf{u}^{\prime}$ in the arguments of $g$ are solutions of a gas particle's equations of motion in Cartesian coordinates with gravity:

$\frac{\mathrm{d} \mathbf{x}^{\prime}}{\mathrm{d} t^{\prime}}=\mathbf{u}^{\prime} ; \quad \frac{\mathrm{d} \mathbf{u}^{\prime}}{\mathrm{d} t^{\prime}}=-\frac{\mathrm{d} \Phi}{\mathrm{d} \mathbf{x}^{\prime}}$

with the final conditions $\mathbf{x}^{\prime}=\mathbf{x}_{\mathrm{f}}$ and $\mathbf{u}^{\prime}=\mathbf{u}$ at $t^{\prime}=t$. To first order in time the solutions of these equations are:

$\mathbf{x}^{\prime}=\mathbf{x}_{\mathrm{f}}-\mathbf{u}\left(t-t^{\prime}\right) ; \quad \mathbf{u}^{\prime}=\mathbf{u}-\left(-\frac{\mathrm{d} \Phi}{\mathrm{d} \mathbf{x}}\right)_{t^{\prime}=t}\left(t-t^{\prime}\right)$

These equations define a single trajectory for the particles which arrive at $\mathbf{x}_{\mathrm{f}}$ at time $t$ with velocity $\mathbf{u}$. The trajectory would generally be curved if we carried terms of higher order than those shown. Even when we are formulating the BGK scheme for a 1-dimensional Cartesian grid there is no escape from considering $\mathbf{u}$ a vector in three dimensions. Both $f$ and $g$ always depend on all of the "molecular" velocity components because individual particles can have velocities of any speed and in any direction. A consequence of this is that when fluxes are eventually computed at $\mathbf{x}_{\mathrm{f}}, f\left(\mathbf{x}_{\mathrm{f}}, \mathbf{u}, t\right)$ is integrated over the full and continuous range of velocities, $\mathbf{u}$, from $-\infty$ to $+\infty$. Therefore fluxes computed from the true distribution function $f\left(\mathbf{x}_{\mathrm{f}}, \mathbf{u}, t\right)$ arise by a weighted integration of the equilibrium distribution function $g\left(\mathbf{x}^{\prime}, \mathbf{u}^{\prime}, t^{\prime}\right)$ not just over one trajectory but over all possible trajectories (in 6-dimensional phase space) which arrive at $\left(\mathbf{x}_{\mathrm{f}}, t\right)$. (We leave it to Sect. 2.6 for a discussion of the implications of this.) For a 1-dimensional problem in Cartesian coordinates all we require is that the trajectory wind up on the wall located at $\left(x_{\mathrm{f}}, y_{\mathrm{f}}, z_{\mathrm{f}}\right)$ where $y_{\mathrm{f}}$ and $z_{\mathrm{f}}$ are fixed for all $x$ because the only non-trivial dimension is the $x$-dimension.

Now as already stated in the introduction, $g$ is not known a priori along all the trajectories passing through $\mathbf{x}_{\mathrm{f}}$ at time $t$. Physically $g$ must describe an equilibrium state so it is sensible to assume a Maxwellian form for $g$ :

$g\left(\mathbf{x}^{\prime}, \mathbf{u}^{\prime}, t^{\prime}\right)=\rho\left(\frac{\lambda}{\pi}\right)^{(K+3) / 2} \mathrm{e}^{-\lambda\left(\left(\mathbf{u}^{\prime}-\mathbf{U}\right)^{2}+\xi^{2}\right)}$.

Here $\mathbf{U}=(U, V, W)$ are the mean macroscopic velocities in the $x, y$ and $z$ directions respectively. To explain why $\mathbf{U}$ has this physical interpretation we go to the onedimensional problem. In one-dimension, $V$ and $W$ are zero 
so $g$ is effectively left with 3 parameters: $\rho, U$ and $\lambda$. For a perfect gas with $K=2 /(\gamma-1)-D$ the "compatability" condition gives an explicit relationship in closed form between the mass, momentum and energy densities and $g$ 's parameters:

$$
\left(\begin{array}{c}
\rho \\
P_{x} \\
\mathcal{E}_{\text {kin }}+\mathcal{E}_{\text {int }}
\end{array}\right)=\left(\begin{array}{c}
\rho \\
\rho U \\
\frac{\rho}{2}\left(U^{2}+\frac{(K+3)}{2 \lambda}\right)
\end{array}\right)
$$

(Note that when $g$ is Maxwellian its velocity moments follow simple recurrence relations (see appendix in $\mathrm{Xu}$ et al. 1996).) Equation (7) assigns physical meaning to $g$ 's parameters: $\rho$ is the mass density, $U$ is the mean macroscopic velocity in the $x$ direction, and $\lambda$ is related to the mass density, $\rho$, and the internal energy density, $\mathcal{E}_{\text {int }}$, in the following way:

$$
\lambda=\frac{(K+3)}{4} \frac{\rho}{\mathcal{E}_{\text {int }}}
$$

where $K$ was defined earlier as the dimension of the vector $\xi . \lambda$ is therefore inversely proportional to the temperature of the gas. Of course mass densities, mean macroscopic velocities and internal energy densities change with space and time in the fluid and their evolution is assumed to be governed by the evolving distribution function $f$. Hence there is a mutual connection between $f$ and $g$. By substituting the formal solution for $f$ (Eq. 4) which itself depends on $g$ into the "compatability condition" on the moments of $g$, one arrives at a set of coupled non-linear integral equations for the space and time dependence of $g$ 's parameters:

$\int \psi_{\alpha} \mathrm{e}^{-\chi_{\alpha} \psi_{\alpha}(\mathbf{r}, t)} \mathrm{d} \Xi=\frac{1}{\tau} \int \psi_{\alpha} \int_{0}^{t} \mathrm{e}^{-\chi_{\beta} \psi_{\beta}\left(\mathbf{r}^{\prime}, t^{\prime}\right)-\frac{\left(t-t^{\prime}\right)}{\tau}} \mathrm{d} t^{\prime} \mathrm{d} \Xi$ $+\mathrm{e}^{-t / \tau} \int \psi_{\alpha} f_{0}\left(\mathbf{x}_{\mathrm{f}}-\mathbf{u} t, t_{0}\right) \mathrm{d} \Xi$

Here we have adopted the following abbreviated notation for $g$ and its parameters. Let

$g=\mathrm{e}^{-\chi_{\alpha} \psi_{\alpha}}$

where $\alpha=1, \ldots, 5$ and

$\psi_{\alpha}=\left(1, \mathbf{u}^{\prime}, \frac{1}{2}\left(\mathbf{u}^{\prime 2}+\xi^{2}\right)\right)$.

We define the transpose of $\psi_{\alpha}$ to be $\psi_{\alpha}{ }^{\mathrm{T}}$ and $\chi_{\alpha}$ to be a vector of $g$ 's parameters:

$\chi_{\alpha}=\left(-\ln \left[\rho(\lambda / \pi)^{(K+3) / 2}\right],-2 \lambda U,-2 \lambda V,-2 \lambda W, 2 \lambda\right)$.

Notice that $\psi_{\alpha}$ has no space dependence whereas $\chi_{\alpha}$ has no velocity dependence. In Eq. (8) we assume that the collision time, $\tau$, which is a function of macroscopic densities is locally constant and hence can be removed outside the integral which is performed over the velocity moments. The computation of $\tau$ is discussed in Sect. 2.3.2. Because $f_{0}$ in the formal solution for $f$ (Eq. (4)) may be straighforwardly constructed from the initial conditions, and because the integral over $g\left(\mathbf{x}^{\prime}, \mathbf{u}^{\prime}, t^{\prime}\right)$ in the formal solution for $f$ is readily evaluated once $g\left(\mathbf{x}^{\prime}, \mathbf{u}^{\prime}, t^{\prime}\right)$ is known, the bulk of the numerical work in the BGK scheme lies in solving the above coupled non-linear integral equations for $g\left(\mathbf{x}^{\prime}, \mathbf{u}^{\prime}, t^{\prime}\right)$.

\subsection{Numerical computation of $f$}

Following the sketch of what is required for a computation of $f$, we outline some aspects of the numerical procedure, particularly those which are relevant to adding gravity to the scheme. The details of the flux computation for the 2D Cartesian case without gravity are presented in $\mathrm{Xu}$ \& Prendergast (1994); Xu et al. (1996); Kim \& Jameson (1998). In Cartesian coordinates we divide the computational domain into cells. We then suppose that we are given the values of mass, momentum and energy densities within each cell at the beginning of a timestep, $t_{0}$. We describe the procedure for computing $f$ at a point $\mathbf{x}_{\mathrm{f}}$ on a cell boundary.

\subsubsection{Numerical computation of $g$}

We begin the numerical computation of the true distribution function $f\left(\mathbf{x}_{\mathrm{f}}, \mathbf{u}, t\right)$ at $\mathbf{x}_{\mathrm{f}}$ by the construction of $g\left(\mathbf{x}^{\prime}, \mathbf{u}^{\prime}, t^{\prime}\right)$ which appears in the integrand of (4). Because we only need to know $f$ at position $\mathbf{x}_{\mathrm{f}}$ located on a cell interface and for the duration of a CFL timestep we do not compute $g\left(\mathbf{x}^{\prime}, \mathbf{u}^{\prime}, t^{\prime}\right)$ precisely at each point along all the trajectories crossing $\mathbf{x}_{\mathrm{f}}$ at $t$. Instead we compute $g$ at the point $\mathbf{x}_{\mathrm{f}}$ on the cell interface at time $t_{0}$ (where $t_{0}$ corresponds to the beginning of a time step) and then approximate it in the spatial and temporal vicinity of $\left(\mathbf{x}_{\mathbf{f}}, t_{0}\right)$ through a Taylor expansion.

$g\left(\mathbf{x}^{\prime}, \mathbf{u}^{\prime}, t^{\prime}\right)=$

$g\left(\mathbf{x}_{\mathrm{f}}, \mathbf{u}, t_{0}\right)\left(1+\frac{\partial \ln g}{\partial \mathbf{x}^{\prime}} \cdot\left(\mathbf{x}^{\prime}-\mathbf{x}_{\mathrm{f}}\right)+\frac{\partial \ln g}{\partial t^{\prime}} \cdot\left(t^{\prime}-t_{0}\right)+\ldots\right)$.

To show the velocity dependence in the spatial and time derivatives of $g$ we rewrite the Taylor series expansion as: $g\left(\mathbf{x}^{\prime}, \mathbf{u}^{\prime}, t^{\prime}\right)=g\left(\mathbf{x}_{\mathrm{f}}, \mathbf{u}, t_{0}\right)\left(1+\mathbf{a} \cdot\left(\mathbf{x}^{\prime}-\mathbf{x}_{\mathrm{f}}\right)\right.$

$$
\left.+\hat{\mathbf{A}} \cdot\left(t^{\prime}-t_{0}\right)\right) \text {. }
$$

Here the vectors a and $\hat{\mathbf{A}}$ depend on the derivatives of the parameters of $g$ with respect to space and time and on $\mathbf{u}$ and $\xi^{2}$ as well. The spatial and time derivatives of $g$ are assumed to be locally constant and we group them as coefficients of particle velocities, $\mathbf{u}^{\prime}, \xi$ in the following way:

$$
\begin{aligned}
& \mathbf{a}=a_{1}+a_{2} u^{\prime}+a_{3} v^{\prime}+a_{4} w^{\prime}+a_{5}\left(\mathbf{u}^{\prime 2}+\xi^{2}\right) \\
& \hat{\mathbf{A}}=\hat{A}_{1}+\hat{A}_{2} u^{\prime}+\hat{A}_{3} v^{\prime}+\hat{A}_{4} w^{\prime}+\hat{A}_{5}\left(\mathbf{u}^{\prime 2}+\xi^{2}\right) .
\end{aligned}
$$

The subscripts $1,2,3,4$, and 5 refer to the five parameters in $g$ (cf. Eq. (8)). Recall that $\mathbf{x}^{\prime}$ and $\mathbf{u}^{\prime}$ lie on curved trajectories (Eq. (5)) if the trajectory is sufficiently prolonged. However if we substitute the expressions for $\mathbf{x}^{\prime}, \mathbf{u}^{\prime}$ given in (5) into the expansion for $g\left(\mathbf{x}^{\prime}, \mathbf{u}^{\prime}, t^{\prime}\right)$ (Eq. (10)) (with the expressions for $\mathbf{a}$ and $\hat{\mathbf{A}}$ given in (11)) and if we retain only terms linear in time, the curvature terms in the particle trajectories do not contribute to the expansion. Hence to first order in time, the solution of the 
BGK equation for the true distribution function $f\left(\mathbf{x}_{\mathrm{f}}, \mathbf{u}, t\right)$ in one-dimensional Cartesian coordinates with gravity is equivalent to the solution for the one dimensional BGK equation in Cartesian coordinates and without gravity. This result generalizes to the case of non-Cartesian geometries. It is the first important result concerning the incorporation of gravity into the BGK scheme.

Our strategy for solving for the terms in the Taylor expansion of $g$ is:

(a) solve for $g\left(\mathbf{x}_{\mathrm{f}}, \mathbf{u}, t_{0}\right)$ and the first spatial derivatives of $g$ from initial conditions. This is possible because as we already showed in Sect. 2.2 there is a direct connection between $g$ 's parameters and the mass, momentum and energy densities which are specified within each cell at the beginning of a timestep $t_{0}$. We can easily obtain the values of the macroscopic densities and their first spatial derivatives with respect to $\mathbf{x}^{\prime}$ at $\left(\mathbf{x}_{\mathrm{f}}, t_{0}\right)$ through interpolation and thereby solve for $g$ and $g$ 's first spatial derivatives at the cell boundary, $\mathbf{x}_{\mathrm{f}}$. We refer to Prendergast \& Xu (1993) for the details of the numerical interpolation including a discussion of interpolation switches. The latter are devices to prevent spurious maxima and other artefacts, and are commonly used in the reconstruction phase in many schemes.

(b) before solving for the time derivatives of $g$, we construct the initial distribution function, $f_{0}$. Because the distribution function $f_{0}$ should reflect a possible nonequilibrium initial state across the cell interface located at position $\mathbf{x}_{\mathrm{f}}$, we assume $f_{0}$ to be composed of two half Maxwellians. It is easiest to see what we mean by this by again considering a one-dimensional Cartesian problem. If we divide the computational domain into intervals whose boundaries are specified by $x_{1}, x_{2}, \ldots, x_{n}$ and we look at flux transfers in the $x$-direction through one of these boundaries located at $x_{\mathrm{f}}$ then we may construct $f_{0}$ at $x_{\mathrm{f}}$ by constructing one half-Maxwellian to the left of $x_{\mathrm{f}}$ and another half-Maxwellian to the right of $x_{\mathrm{f}}$. The parameters of the two half Maxwellians as well as their slopes are obtained from the initial conditions, just as $g$ 's parameters were obtained but the left (right) Maxwellian is computed from the mass, momentum and energy densities interpolated from the left (right) side of the cell interface. This choice for the form of $f_{0}$ is not unique. The details of $f_{0}$ should not matter, since they are rapidly damped for small $\tau$.

(c) Finally we solve for the time derivatives of $g$ by insisting that the "compatability condition" is satisfied on average over the CFL time interval. Due to our approximate computation of $g$ and hence of $f, g$ and $f$ cannot have exactly the same moments for the duration of a CFL time step over all the trajectories arriving at $\mathbf{x}_{\mathrm{f}}$. However we insist that they have the same moments at $\mathbf{x}_{\mathrm{f}}$ and on average over the time interval $0 \leq t^{\prime} \leq t$ for which the true distribution function is computed.

$\int_{0}^{t} \int \psi_{\alpha}^{\mathrm{T}}\left(f\left(\mathbf{x}_{\mathrm{f}}, \mathbf{u}, t^{\prime}\right)-g\left(\mathbf{x}_{\mathrm{f}}, \mathbf{u}, t^{\prime}\right)\right) \mathrm{d} \Xi \mathrm{d} t^{\prime}=0$.
This condition gives an expression for $\hat{\mathbf{A}}$ (see $\mathrm{Xu} \&$ Prendergast 1994). It is worth remarking that the manner in which the time derivatives of $g$ are computed is in some sense implicit. Because Eq. (12) is applied over the entire time interval, expressions for $\hat{\mathbf{A}}$ arise from information throughout the entire time interval as opposed to just information at the beginning of a time step. We also point out that by finding the time-dependence of $f$ through the formal solution for $f$ plus the "compatability condition" applied on average over an updating time step, Prendergast \& Xu's (1993) BGK scheme bypasses the stiffness in the BGK equation which would force the updating timestep to be the particle collision timescale $\tau$. Because the updating time step is determined from the CLF condition hydrodynamics may be performed with the BGK scheme. Earlier attempts at implementing the BGK equation (e.g. Chu 1965) were constrained by the stiffness of the BGK equation and their application was therefore limited to rarefied gas dynamics.

\subsubsection{The collision time, $\tau$}

Aside from the Courant factor in the computation of the CFL time step (Sect. 2.5) (and certain constants which depend on the adopted interpolation rules), the expression for the collision time, $\tau$, contains the only 2 parameters in the code. According to gas kinetic theory, the mean time between collisions in a gas with density $\rho$ and temperature $T$ is proportional to $1 /\left(\rho T^{1 / 2}\right)$. Since $\lambda \approx 1 / T$, $\tau=\mathcal{C}_{1} \sqrt{\lambda} / \rho$ where $\mathcal{C}_{1}$ is a proportionality constant and the first of the two parameters in $\tau$. We add a second term to the expression for the collision time. In one-dimensional Cartesian coordinates this second term is:

$$
\mathcal{C}_{2} \frac{\left|\sqrt{\lambda_{l}} / \rho_{l}-\sqrt{\lambda_{r}} / \rho_{r}\right|}{\sqrt{\lambda_{l}} / \rho_{l}+\sqrt{\lambda_{r}} / \rho_{r}} \frac{\left|p_{l}-p_{r}\right|}{\left(p_{l}+p_{r}\right)}
$$

The subscript $l(r)$ denotes quantities interpolated from the left (right) side of the cell interface at which we compute the collision time $\tau$ and $p$ is the gas pressure. We choose the above form for the second term in the expression for the collision time $\tau$ because it helps with shocks across which there are gradients in $p$. As stated in the introduction (Sect. 1), we motivate this second term in the collision time as follows: given that the coefficients of heat conduction and viscosity are proportional to the collision time $\tau$, the second term in the expression for $\tau$ acts to increase both heat conduction and viscosity at shock fronts but not at contact discontinuities across which pressure is continuous. When the second term in the collision time dominates, the order of the scheme is reduced and the true distribution function $f$ is determined more by the initial distribution function $f_{0}$ than by the integral over $g$. 


\subsection{Computing the total fluxes}

Once $f\left(\mathbf{x}_{\mathbf{f}}, t\right)$ is fully known as a function of the parameters of $g, f_{0}$, the collision time $\tau$, and $t$, the time-dependent fluxes at $\mathbf{x}_{\mathrm{f}}$ can be computed. We illustrate flux computation for a one-dimensional Cartesian problem. In that case we are interested only in the $x$-component of the total flux, i.e. the component of the flux which is perpendicular to a cell wall at $x_{\mathrm{f}}$. The total amount of mass, $x$-momentum and energy densities transferred through the wall perpendicular to the $x$-direction in a CFL time step $0 \leq t \leq T$ is found from:

$\left(\begin{array}{c}\mathcal{F}^{\rho} \\ \mathcal{F}^{P_{x}} \\ \mathcal{F}^{\mathcal{E}_{\text {kin }}+\mathcal{E}_{\text {int }}}\end{array}\right)=$

$\int_{0}^{T} \int u_{x}\left(\begin{array}{c}1 \\ u_{x} \\ \frac{1}{2}\left(u_{x}^{2}+\xi^{2}\right)\end{array}\right) f\left(x_{\mathrm{f}}, \mathbf{u}, t\right) \mathrm{d} \Xi \mathrm{d} t$.

Note that in the end, the gas kinetic origin of the expressions for the fluxes disappears and they are written as highly non-linear functions of the mass, momenta and energy densities which are presumed known at the beginning of the time step.

\subsection{Courant time step}

The time step $T$ used to evolve the dynamical equations is chosen so that it satisfies the CFL condition. Physically the CFL condition limits the distance that information can travel in one time step to one cell. For the onedimensional Cartesian case we compute $T$ for each cell on the grid from

$$
\begin{array}{r}
a_{i+1 / 2} T^{2}+ \\
\left(\left|U_{i+1 / 2}\right|+c_{i+1 / 2}\right) T+\mathcal{L}_{i+1 / 2}=0
\end{array}
$$

where $a$ is the local gravitational acceleration due to gravity, i.e. $-\frac{\partial \Phi}{\partial x}, U$ is the local macroscopic velocity, $c$ is the local adiabatic sound speed and

$\mathcal{L}_{i+1 / 2}=\min \left(\left(x_{i+1}-x_{i}\right),\left(x_{i}-x_{i-1}\right),\left(x_{i-1}-x_{i-2}\right)\right)$.

This choice for $\mathcal{L}_{i+1 / 2}$ insures that flux transport between adjacent cells is carried out for a length of time short enough so as not to empty them.

Finally the time step $T$ is chosen from

$T=(1-\epsilon) \min (T)$

where $(1-\epsilon)$ is a safety factor; it is called the CFL factor (typically $\epsilon \approx .6$ ).

\subsection{Comments about flux computation with the BGK method}

Unlike Riemann solvers which propagate information along the characteristics of the Euler equations, the BGK scheme propagates information along the characteristics of the Boltzmann equation. These are particle trajectories in phase space. In the BGK scheme fluid properties along the full continuum of trajectories passing through $(\mathbf{x}, \mathbf{t})$ contribute to the instantaneous fluxes at $(\mathbf{x}, \mathbf{t})$. The implications of this are many. Firstly it bypasses the limitation of lattice Boltzmann codes which discretize velocity space thereby restricting the number of directions for the propagation of information and thereby also limiting the magnitudes of the gas velocities.

Secondly, using all the trajectories with appropriate weighting endows the BGK scheme with intrinsic upwindedness. An upwind scheme computes fluxes using information coming from the same direction as the flow. When computing fluxes, the BGK scheme does not select trajectories corresponding to the direction of flow and discount the rest; instead it uses information carried by all trajectories arriving at the place where we construct $f$, but it weights the information along each trajectory according to the number of particles assigned to the trajectory by the BGK solution. This is what we mean by an intrinsically upwind scheme.

Thirdly, the consideration of the full continuum of trajectories for the flux computation creates the potential for a truly multidimensional code. Because the velocity integration includes all propagation directions and because the trajectories can be followed multidimensionally, the flux in one direction can potentially be computed from information coming with all speeds and all directions. For a genuinely multidimensional scheme, the interpolation of the initial conditions for the reconstruction stage must also be multidimensional. For example, for a computation on a two dimensional Cartesian grid, the reconstruction of the initial conditions at a point $(x, y)$ should include information from cells surrounding the point in both the $x$ and the $y$ directions.

At the present time, the multidimensional BGK schemes which have been implemented do not take advantage of the truly multidimensional possibility that the BGK scheme offers. They still simplify flux updates by separating them into individual operations in independent directions. This is an example of "operator-splitting". On the optimistic side, a truly multidimensional BGK scheme is conceivable. The same cannot be said for schemes based on Riemann solvers. Even in two dimensions it is not feasible to construct a truly multidimensional Riemann solver. Upon representing the initial conditions as piecewise constant in two-dimensional cells, the Riemann solution is straightforward to apply at each of the interfaces of a two-dimensional cell but problematic at cell corners. Furthermore, by propagating information only along the directions normal to cell interfaces, a two-dimensional Riemann solver has the same limitation as an operator-split method: it does not accommodate the possibility that waves in two-dimensional flows may propagate in infinitely many directions (Roe 1986). 


\subsection{The source terms}

Because to first order in time the computation of the true distribution function, $f(x, \mathbf{u}, \mathbf{t})$, and hence the computation of the hydrodynamical fluxes is unaffected by gravity, gravity's influence on the gas flow is relegated to source terms.

\subsubsection{Gravitational momentum source terms}

In one-dimensional Cartesian coordinates gravitational source terms contribute to the momentum change in a cell over a CFL time step $t=0$ to $t=T$ in the following form:

$\mathcal{S}_{P_{x}}\left(x_{i-1 / 2}, T\right)=-\int_{0}^{T} \int_{x_{i-1}}^{x_{i}} \rho\left(x^{\prime}, t^{\prime}\right) \frac{\partial \Phi}{\partial x}\left(x^{\prime}, t^{\prime}\right) \mathrm{d} x^{\prime} \mathrm{d} t^{\prime}$.

This integral may be computed in a number of ways. One possible approach is to do a linear interpolation in $x^{\prime}$ using the values of the mass densities and forces at the cell wells surrounding the cell for which we wish to compute the source term.

The time integration required by (16) takes advantage of the fact that we know the new mass densities at the end of an updating time step, i.e. at $t=T$, before we've completed the updates of the other fluid quantities, i.e. $P_{x}, \mathcal{E}_{\text {kin }}+\mathcal{E}_{\text {int }}+\mathcal{E}_{\text {grav }}$. This is a consequence of there being no source terms in the mass continuity equation. From the mass densities at times $t=0$ and $t=T$ we may perform a bilinear interpolation in $x$ and $t$ for the integral in Eq. (16). Specifically

$$
\begin{array}{r}
\mathcal{S}_{P_{x}}\left(x_{i-1 / 2}, T\right)= \\
-\frac{T}{2} \int_{x_{i-1}}^{x_{i}}\left\{\rho\left(x_{i-1}, 0\right) \frac{\partial \Phi}{\partial x}\left(x_{i-1}, 0\right)\left(1-\beta\left(x^{\prime}\right)\right)+\right. \\
\rho\left(x_{i}, 0\right) \frac{\partial \Phi}{\partial x}\left(x_{i}, 0\right)\left(\beta\left(x^{\prime}\right)\right)+ \\
\rho\left(x_{i-1}, T\right) \frac{\partial \Phi}{\partial x}\left(x_{i-1}, T\right)\left(1-\beta\left(x^{\prime}\right)\right)+ \\
\left.\rho\left(x_{i}, T\right) \frac{\partial \Phi}{\partial x}\left(x_{i}, T\right)\left(\beta\left(x^{\prime}\right)\right)\right\} \mathrm{d} x^{\prime}
\end{array}
$$

where

$\beta\left(x^{\prime}\right)=\frac{\left(x^{\prime}-x_{i}\right)}{\left(x_{i+1}-x_{i}\right)}$.

\subsubsection{The energy equation}

As for gravity's contribution to the energy equation, we explore two versions of it. One form of the energy equation:

$\frac{\partial}{\partial t}\left(\mathcal{E}_{\text {kin }}+\mathcal{E}_{\text {int }}\right)+\frac{\partial}{\partial x}\left(F^{\mathcal{E}_{\text {kin }}+\mathcal{E}_{\text {int }}}\right)-P_{x} \frac{\partial \Phi}{\partial x}=0$

has a gravitational energy source term, $-P_{x} \frac{\partial \Phi}{\partial x}$. The gravitational energy source term may be computed analogously

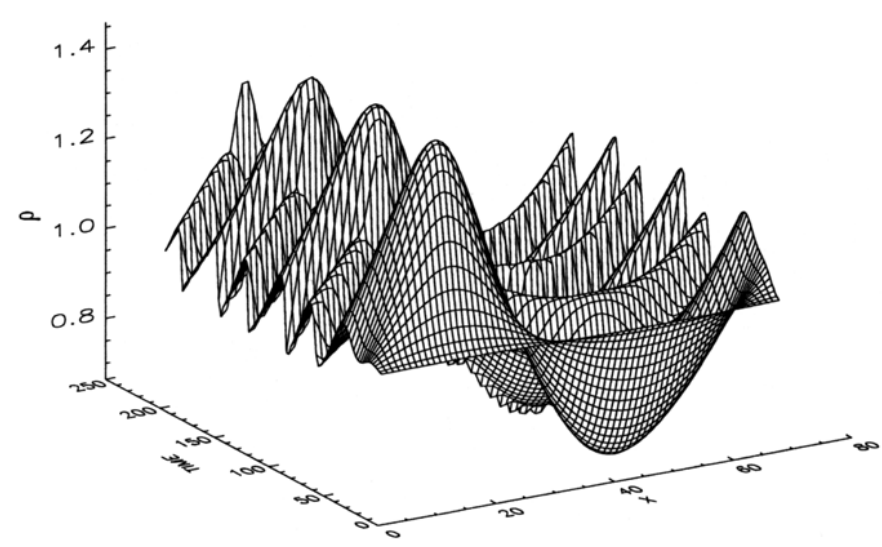

Fig. 1. Early stage of time evolution of mass density profile for run with total energy conserving (ECS) scheme, including gravitational energy. Total time 201.19 ( $\equiv 3.3$ sound crossing times)

to the way in which the gravitational momentum source terms are computed (cf. Eq. (17)).

As mentioned in Sect. 2.1, another form of the energy equation incorporates the gravitational energy source term into the energy fluxes and hence is in conservative form:

$\frac{\partial}{\partial t}\left(\mathcal{E}_{\text {kin }}+\mathcal{E}_{\text {int }}+\mathcal{E}_{\text {grav }}\right)+\frac{\partial}{\partial x}\left(F^{\mathcal{E}_{\text {kin }}+\mathcal{E}_{\text {int }}}+\Phi F^{\rho}\right)=0$.

The conservative form of the energy equation requires a good computation of the gravitational energy because now it, in addition to the kinetic energy, $\mathcal{E}_{\text {kin }}$, must be subtracted from the total energy for the purpose of obtaining the internal energy, $\mathcal{E}_{\text {int }}$. These subtractions are potentially extremely delicate. They are important because the internal energy is used in computing $\lambda$ (which is inversely proportional to the temperature), a crucial parameter for the hydrodynamic fluxes.

Because the form of the gravitational energy is similar to the form of the gravitational source terms,

$\mathcal{E}_{\text {grav }}\left(x_{i-1 / 2}\right)=\int_{x_{i-1}}^{x_{i}} \rho\left(x^{\prime}\right) \Phi\left(x^{\prime}\right) \mathrm{d} x^{\prime}$

it may be numerically computed in the same manner, i.e. by a linear interpolation to the values of the densities and the potentials at the walls bounding a cell.

For the gravitational flux term, $\frac{\partial}{\partial x}\left(\Phi F^{\rho}\right)$, in the conservative energy equation we use the value of the gravitational potential $\Phi$ at the same point $x_{\mathrm{f}}$ at which we construct the true distribution function, $f$, for the other fluxes.

\subsection{Summary of the BGK method with gravity}

We have given a description of the procedure for computing hydrodynamical fluxes and source terms in a BGK 


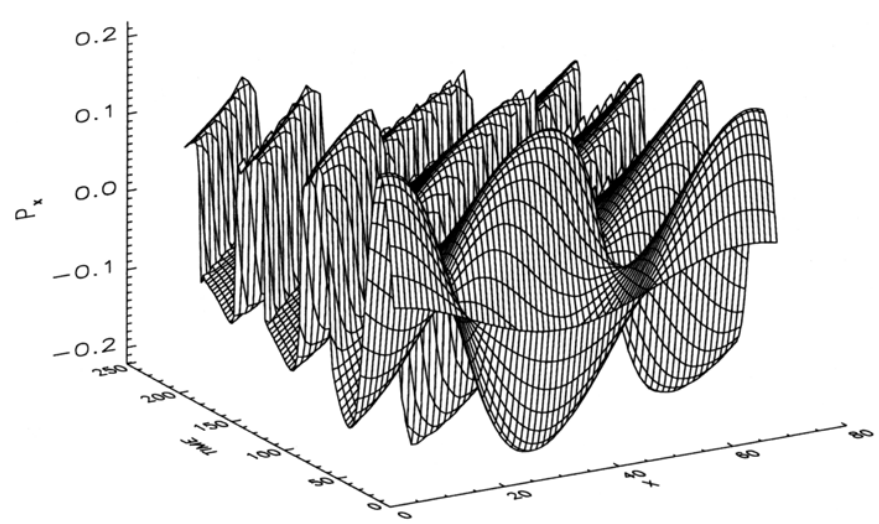

Fig. 2. Early stage of time evolution of $x$-momentum density profile for run with ECS scheme. Total time 201.19 (三 3.3 sound crossing times)

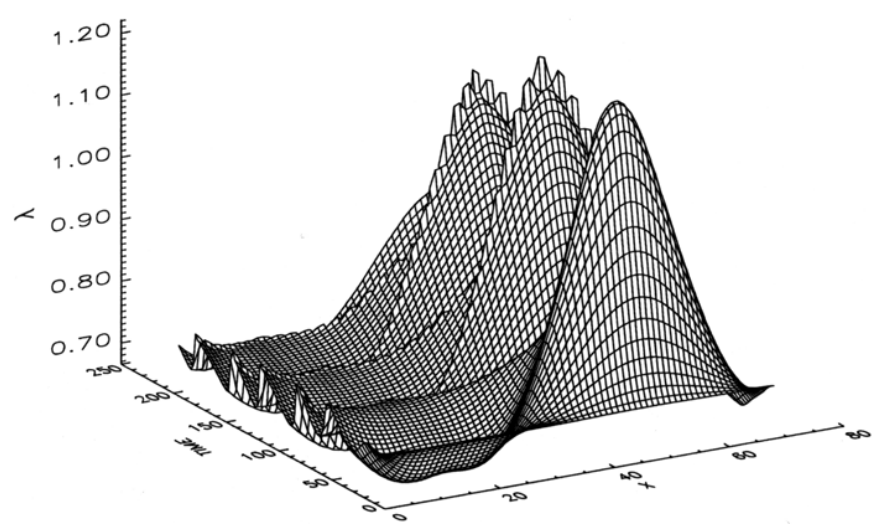

Fig. 3. Early stage of time evolution of $\lambda$ profile for run with ECS scheme. Total time 201.19 ( $\equiv 3.3$ sound crossing times)

scheme designed for hydrodynamics in the presence of a time-independent gravitational field.

1.) While incorporating gravity into the BGK scheme we have found that to first order in time gravity does not alter the computation of the distribution function from which hydrodynamical fluxes are computed. It enters into the hydrodynamical computation through gravitational momentum source terms as well as through either a gravitational energy source term or through gravitational energy fluxes. The latter depends on the chosen form of the energy equation.

2.) The energy source term formulation by definition does not guarantee conservation of total (kinetic + internal + gravitational) energy. An inaccurate computation of the energy source term introduces numerical heating and/or cooling. Because the energy equation can be manipulated into a conservative form for the total kinetic + internal + gravitational energy, computation of an energy source term is avoidable. In lieu of an energy source term, the gravitational energy enters into the hydrodynamical computation through flux terms. These flux terms do not require any flux computation in addition to the computation which is already required in a hydrodynamics scheme

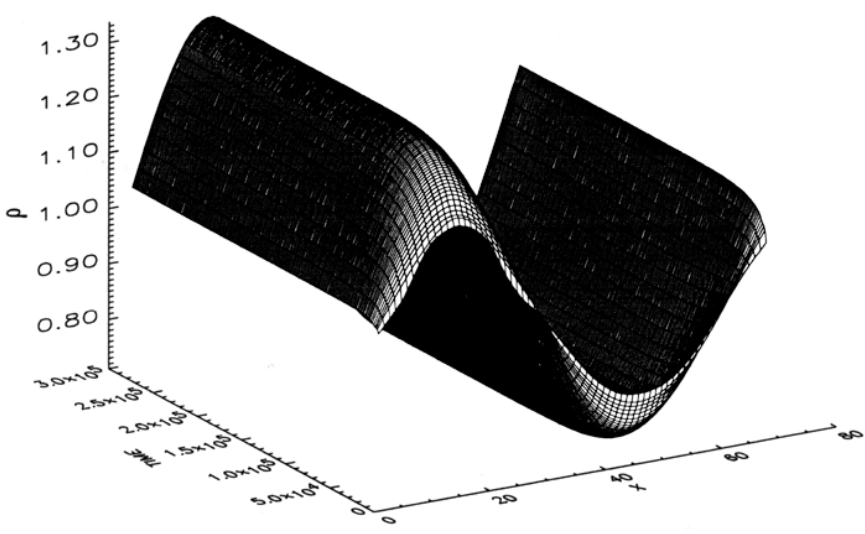

Fig. 4. Mass density profile for long run with ECS scheme after total time of 285739.54 ( $\equiv 4706$ sound crossing times)

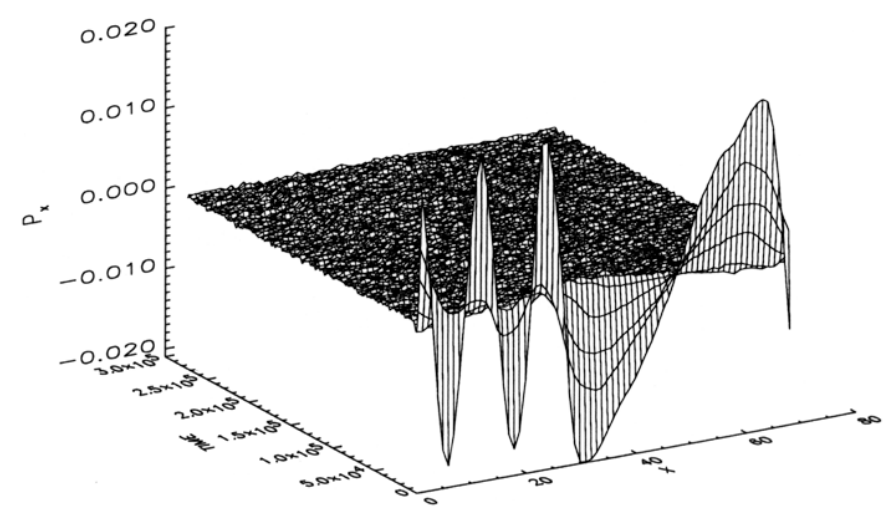

Fig. 5. $X$-momentum density profile for long run with ECS scheme after total time of 285739.54 ( $\equiv 4706$ sound crossing times)

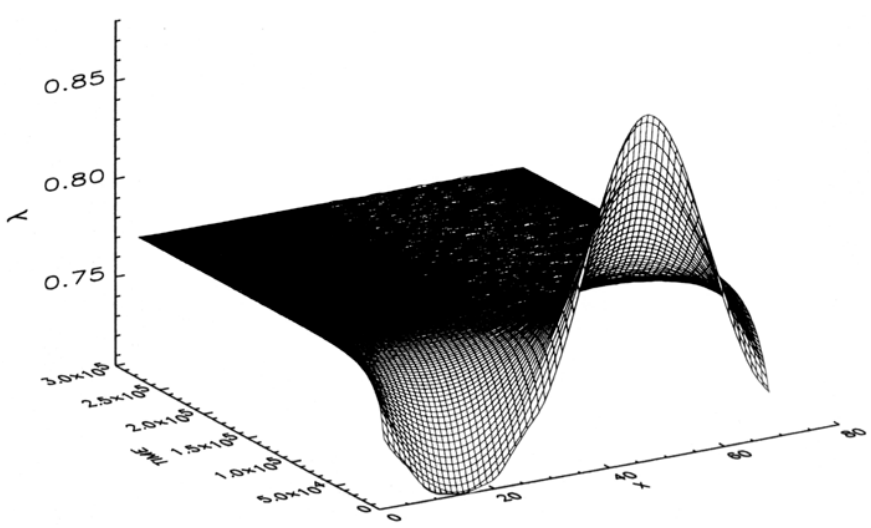

Fig. 6. Lambda profile for long run with ECS scheme after total time of 285739.54 ( $\equiv 4706$ sound crossing times). Note that lambda becomes and remains constant after some time. This is entirely in accord with expectation of the long-term behaviour of a closed system: the temperature (i.e. $\frac{1}{\lambda}$ ) reaches equilibrium and stays there. Compare this result with Fig. 9 which shows long term heating of numerical origin using a different version of the code 


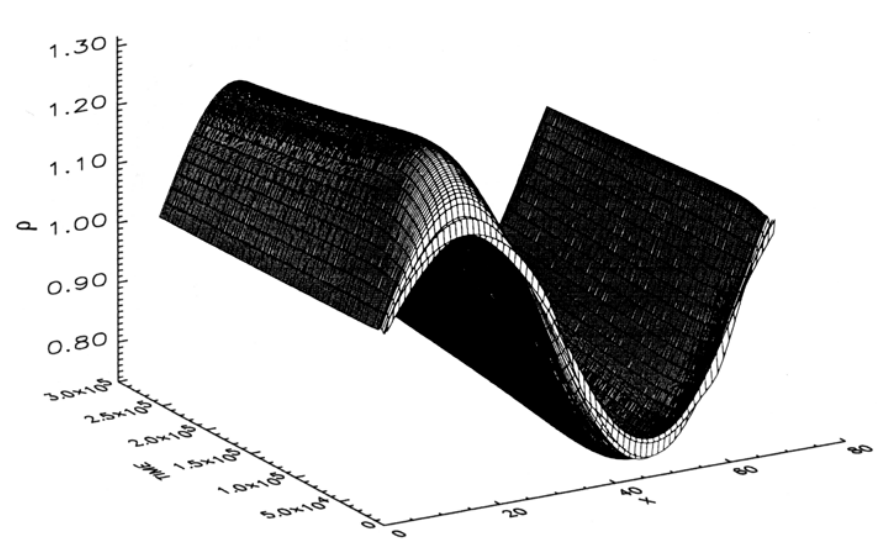

Fig. 7. Mass density profile for long run with energy source term (EST) scheme after total time of 261888.6 ( $\equiv 4313$ sound crossing times). Note that the density profile has not settled to a constant profile with time. Compare with Fig. 4 which uses the ECS scheme

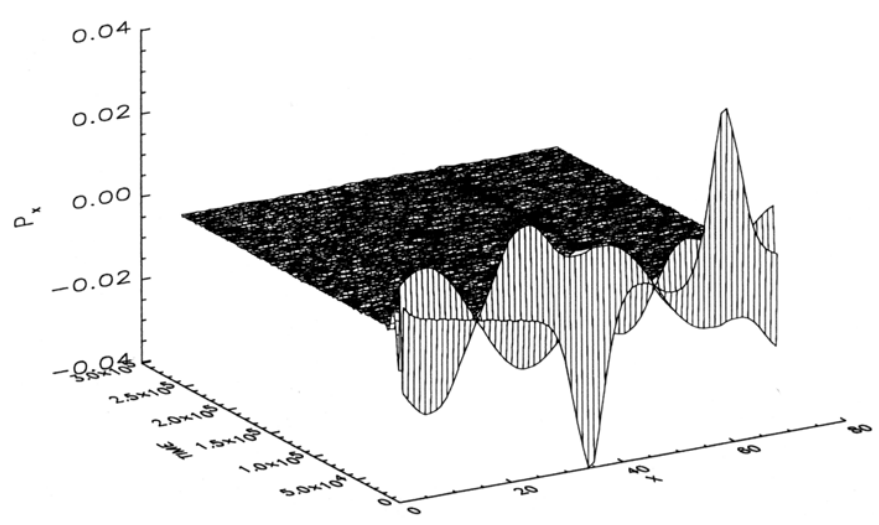

Fig. 8. $X$-momentum density profile for long run with EST scheme after total time of 261888.6 ( $\equiv 4313$ sound crossing times)

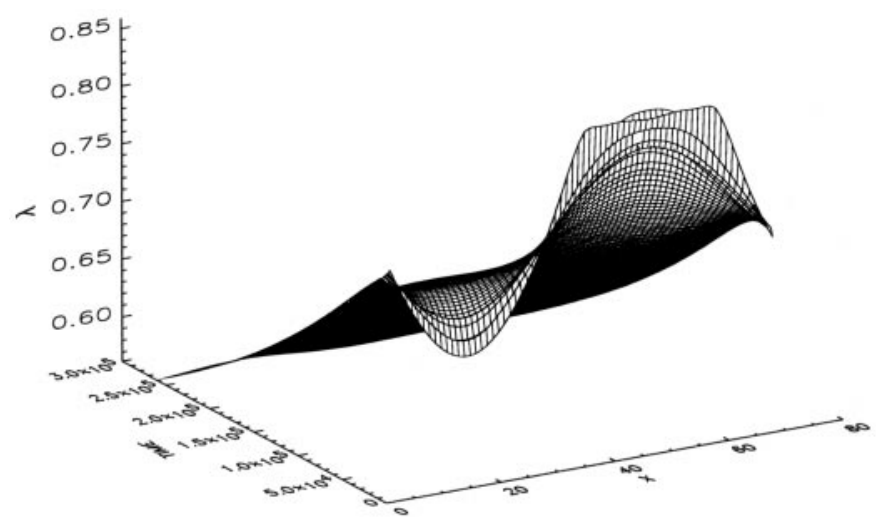

Fig. 9. Lambda profile for long run with EST scheme after total time of 261888.6 ( $\equiv 4313$ sound crossing times). Note that lambda descreases instead of remaining constant (as it should) at large time. The effect is due to numerical heating and is absent in the results from the totally conservative (ECS) scheme (cf. Fig. 6)

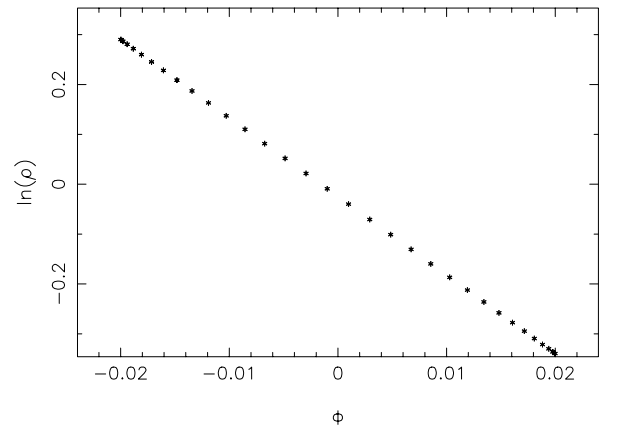

Fig. 10. Check to see how well final density profile from long run with ECS scheme matches the analytic solution for the equilibrium profile. The points should fall on a straight line if there is a perfect match to the analytic solution. See text (Sect. 3.1) for parameters of a least squares fit to the points

without gravity. This is because they are conveniently the product of the gravitational potential and the mass flux terms.

3.) Even though an implementation of the conservative form of the energy equation by definition assures total energy conservation, it introduces another challenge brought on by the inclusion of gravity into a hydrocode. For the calculation of hydrodynamical fluxes in the BGK scheme it is essential to accurately compute the temperature and therefore the internal energy. With gravity and with an implementation of the conservative form of the energy equation it therefore becomes necessary to accurately compute the gravitational energy so that it, in addition to the kinetic energy, may be subtracted from the total energy (kinetic + internal + gravitational) to give the internal energy without introducing numerical heating (or cooling) into the scheme.

\section{Results}

We present results from two tests of the BGK scheme in the presence of a time-independent gravitational potential. The first test reveals the effect on a simulation of using different forms of the energy equation. The second test is performed on an axisymmetric cylindrical grid. Both tests reveal the capability of the BGK scheme with gravity to reach an equilibrium state and to maintain a gas configuration in hydrostatic equilibrium.

\subsection{On a 1D Cartesian grid: Gas falling into a fixed external potential}

We perform this test case to compare the results from two versions of the BGK scheme with gravity: one using the conservative form of the energy equation (the ECS scheme) and one using the energy source term formulation of the energy equation (the EST scheme). The initial 

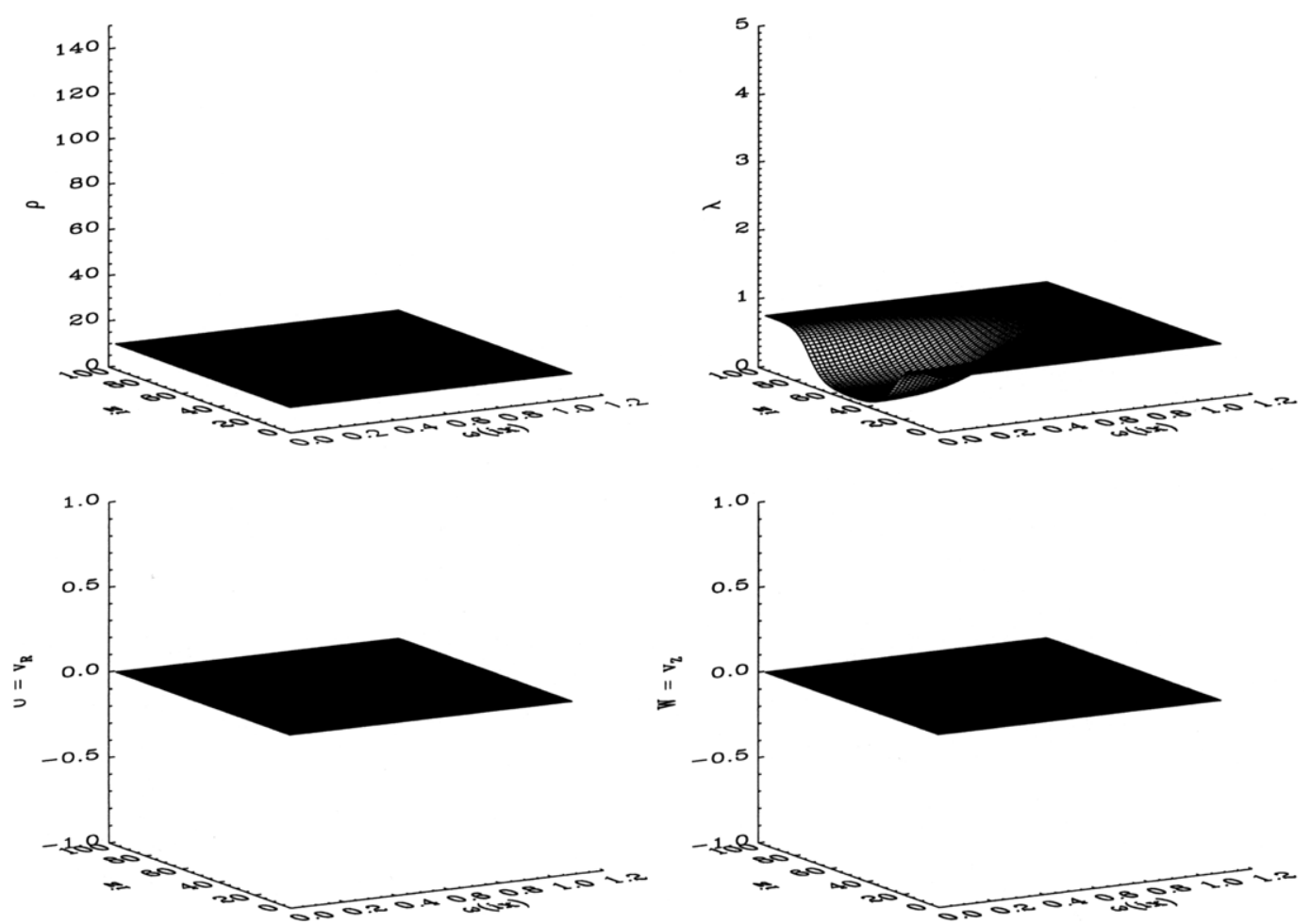

Fig. 11. The initial conditions for the simulation of gas falling into a fixed external spherically symmetric gravitational potential well: uniform density (top left), lambda (top right), zero radial velocity (bottom left) and zero vertical velocity (bottom right)
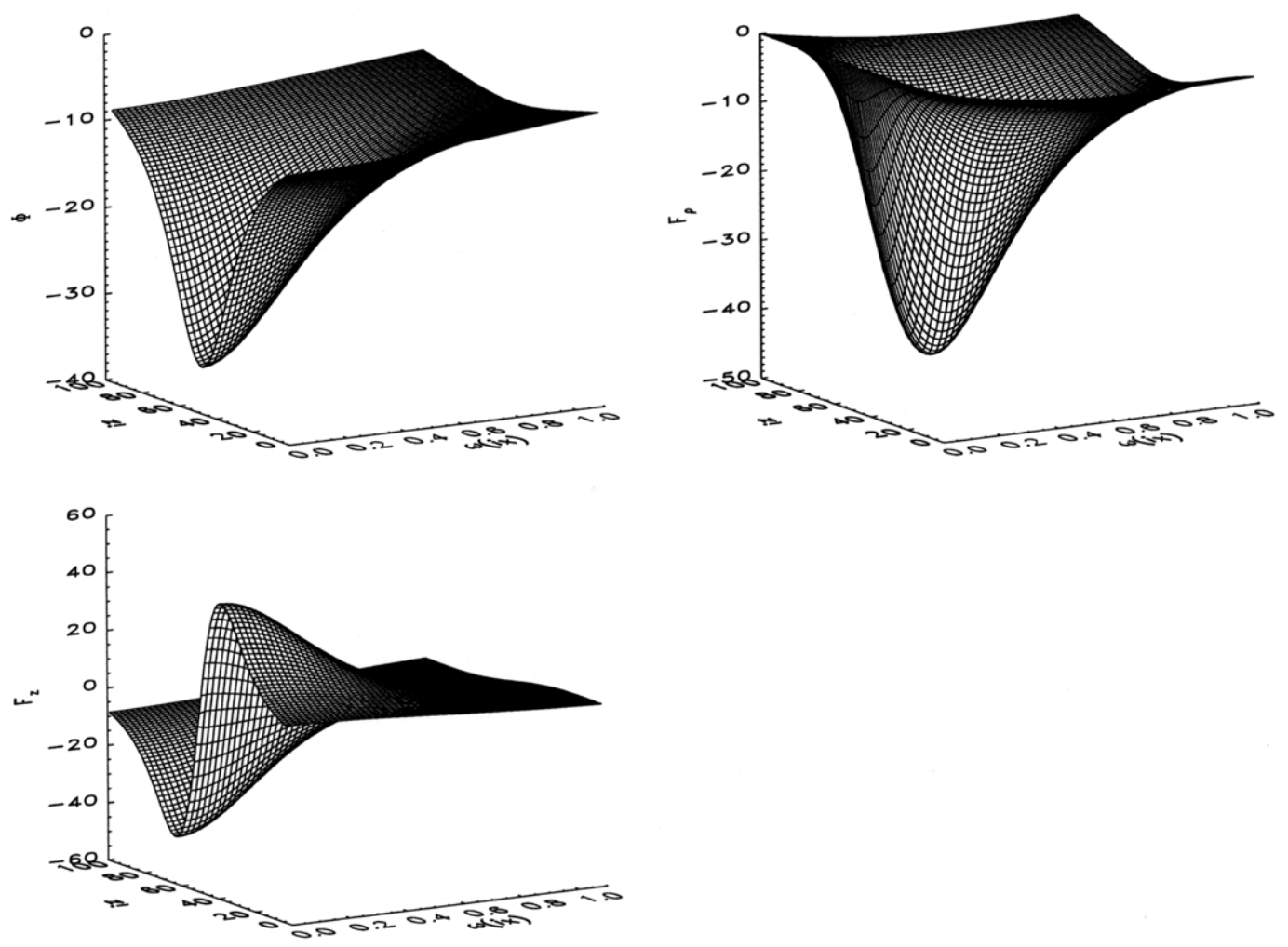

Fig. 12. The gravitational potential (top left), the radial force (top right) and the vertical force (bottom left), each as a function of $\varpi$ and $z$. They are fixed in time and space throughout the entire run 

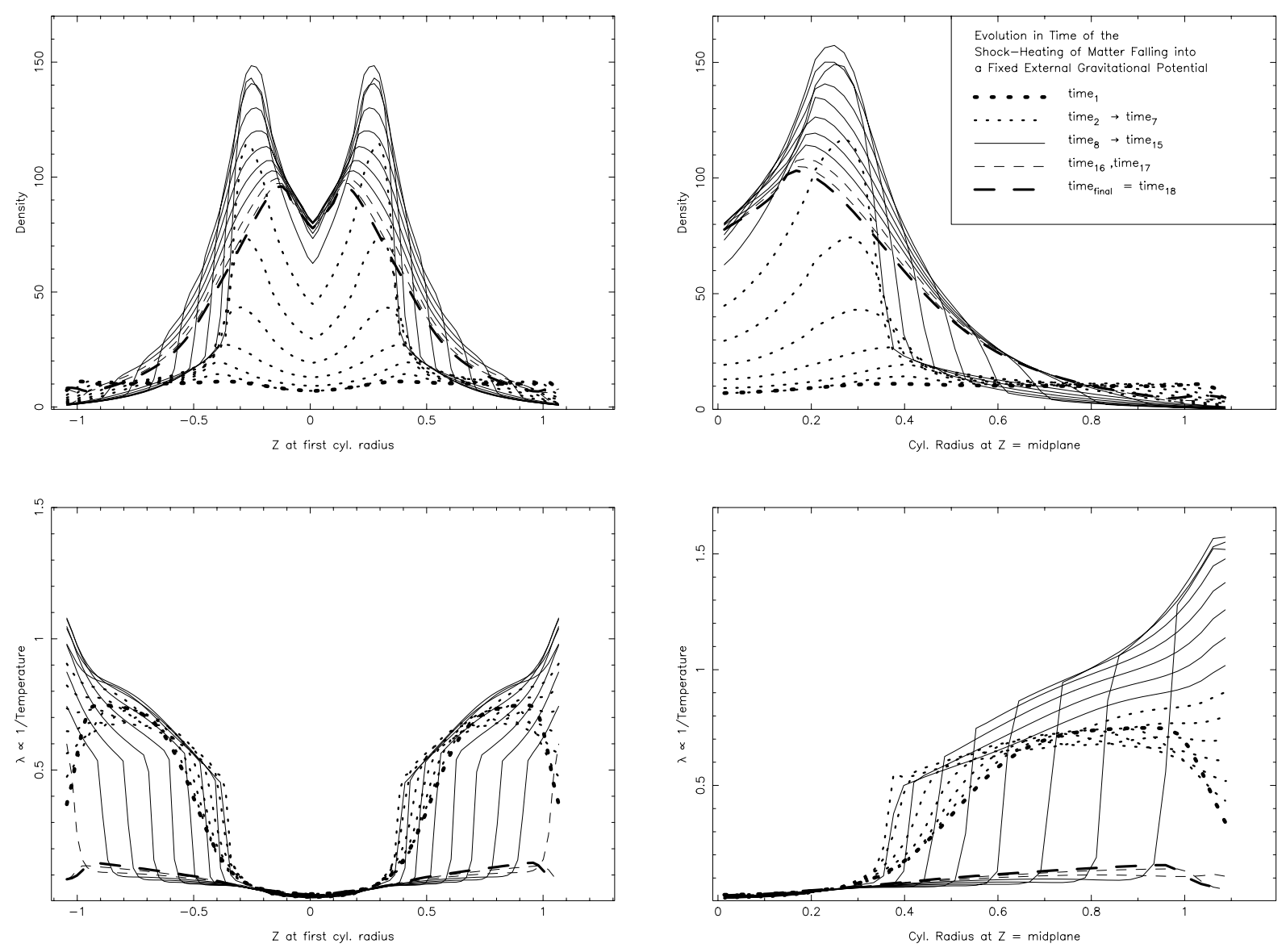

Fig. 13. Results from simulations of the shock heating of gas falling into a fixed external spherically symmetric gravitational potential well. Time evolution of the density (top) and lambda (bottom) along two rays of the cylindrical grid. The cylindrical grid has 50 cells in $\varpi$ and 100 cells in $z$

conditions for the two simulations are identical. Each simulation is performed on a 1D Cartesian grid with 68 evenly spaced cells. The gas is initially stationary $\left(P_{x}=0\right.$, where $P_{x}$ is the $x$-momentum) and homogeneous with mass density $\rho=1$ and internal energy density $\mathcal{E}_{\text {int }}=1$ in each cell. The external gravitational field is constant in time and its potential, $\Phi$ has the form of a sine wave, i.e. $\Phi=-\Phi_{0}(\mathcal{L} /(2 \pi)) \sin (2 \pi x / \mathcal{L})$ with $\Phi_{0}=0.02$ and $\mathcal{L}=64$. Because we apply periodic boundary conditions there are two ghost cells at each end of the grid. We take $\epsilon$ in the Courant condition to be $.4, \gamma=\frac{5}{3}$ and $\mathcal{C}_{1}=.01$ and $\mathcal{C}_{2}=1$ in the expression for the collision time $\tau$. We run both versions of the code for 500000 iterations. The total time at the end of the run with the ECS scheme is 285739.54 in machine units (4706 in units of the initial sound crossing time). Note that we define the total time to be the sum of the lengths of each of the individual CFL timesteps used for the updates. For the run with the EST scheme the total time at the end of the run is 261888.6 in machine units (4313 in units of the initial sound crossing time).

Figures 1 through 6 show results from the version of the BGK scheme which implements the energy conserving form of the energy equation and which therefore keeps track of the total kinetic + internal + gravitational energy density within each cell. Figures 7 through 9 on the other hand show results from an implementation of the energy equation in a non-conservative form, i.e. one in which gravity is incorporated into the energy equation through a gravitational source term. The plots show the time evolution of various quantities (i.e. mass density, $x$-momentum and lambda) as functions of position on the grid. Because the cells are evenly spaced with a spacing of $\Delta x=1$, we label cell positions by an index $X$ which runs from 1 to 68 in the plots. The quantity on the vertical axis in each of the plots is also plotted at approximately equal time intervals.

For the simulations using the energy conserving scheme we present not only plots showing the long term evolution of the gas, but also some (Figs. 1, 2,3) which show the evolution of the gas early on in the simulation. In these figures, particularly in the $x$-momentum plot (i.e. Fig. 2), we see the steepening of the sound waves into shocks. With the values for the internal energy density and the mass density given in the initial conditions, the initial sound crossing time for the gas is 60.72 (in machine units). For the data presented in Figs. 1, 2 and 3 

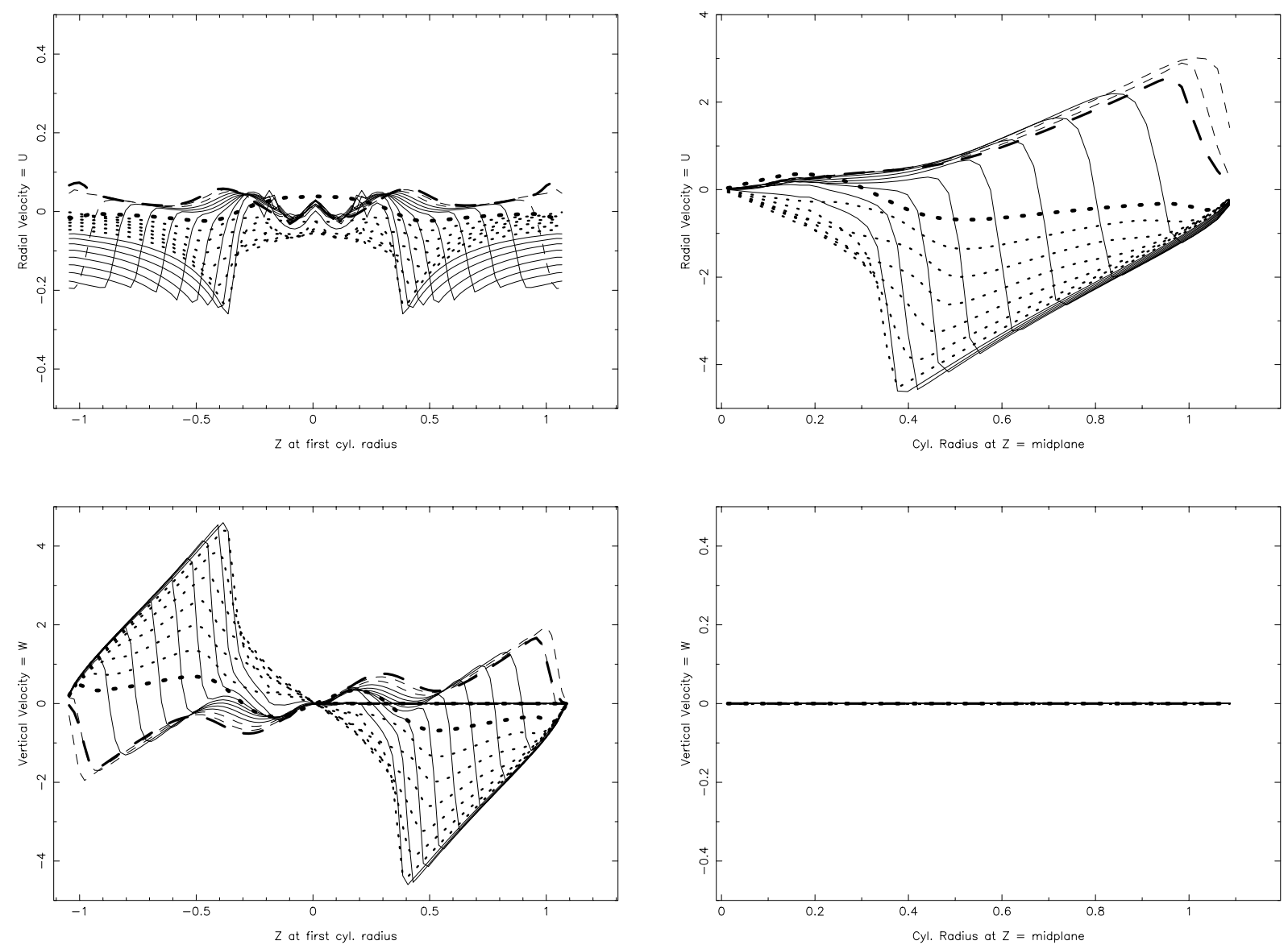

Fig. 14. Time evolution of the radial velocity (top) and vertical velocity (bottom) along two rays of the cylindrical grid (cf. Fig. 13)

the total time elapsed is 201.19 (in machine units). This is equivalent to 3.3 sound crossing times and indeed we see that the gas has undergone a bit more than three oscillations in these figures.

In the plots showing the long term evolution of the gas (Figs. 4 to 9), at the time of the earliest curve (time $\approx 2816$ ) the gas has experienced about 46 sound crossing times (we compute this using the value for the initial sound crossing time). The mass density in both the ECS scheme and the EST scheme at $t=2816$ is high at the location of the gravitational potential minumum and low at the location of the potential maximum indicating that the gas has fallen into the potential well. Because the gas should convert some of its gravitational energy into internal energy as it falls into the potential well, we look to see if the gas at the density maximum is hotter. As expected, the temperature at $t=2816$ is highest (hence lambda is lowest) at the location of maximum density and the temperature is lowest (hence lambda is highest) at the location of minimum density. For reference, lambda at the beginning of the simulations is 0.75 .

In the results from the scheme using the nonconservative form of the energy equation, there is a monotonic decline in lambda (Fig. 9) at increasing times. Since lambda is inversely proportional to the temperature, this decline corresponds to a temperature rise which we believe is due to "numerical heating". This heating persists with time and therefore deters the gas from reaching thermodynamic equilibrium. The heating is reflected in the value of the total energy (kinetic + internal + gravitational) summed over the entire grid at the end of the run: it is $\approx 10 \%$ greater than the initial total energy.

In contrast, the results from the run with the energy conserving implementation of the energy equation show a gas which reaches thermodynamic equilibrium: the lambda plot (Fig. 6) is level at late times with a mean value of .77 (deviation $\approx 1.52 D-04$ ) and remains at the same value throughout the entire grid for as long as we run the simulation. The difference between the initial and final total energies on the grid is $4.3451 D-13$. In addition to thermodynamic equilibrium, the gas reaches mechanical equilibrium as verified by the $x$-momentum plot: at the final time plotted $P_{x}$ is $-1.16 D-20$ in the mean with a deviation of $2.04 D-04$. We believe that the noisiness in the final $P_{x}$ state is a consequence of the run being performed without the use of interpolation switches for the flux computation. The analytic solution 

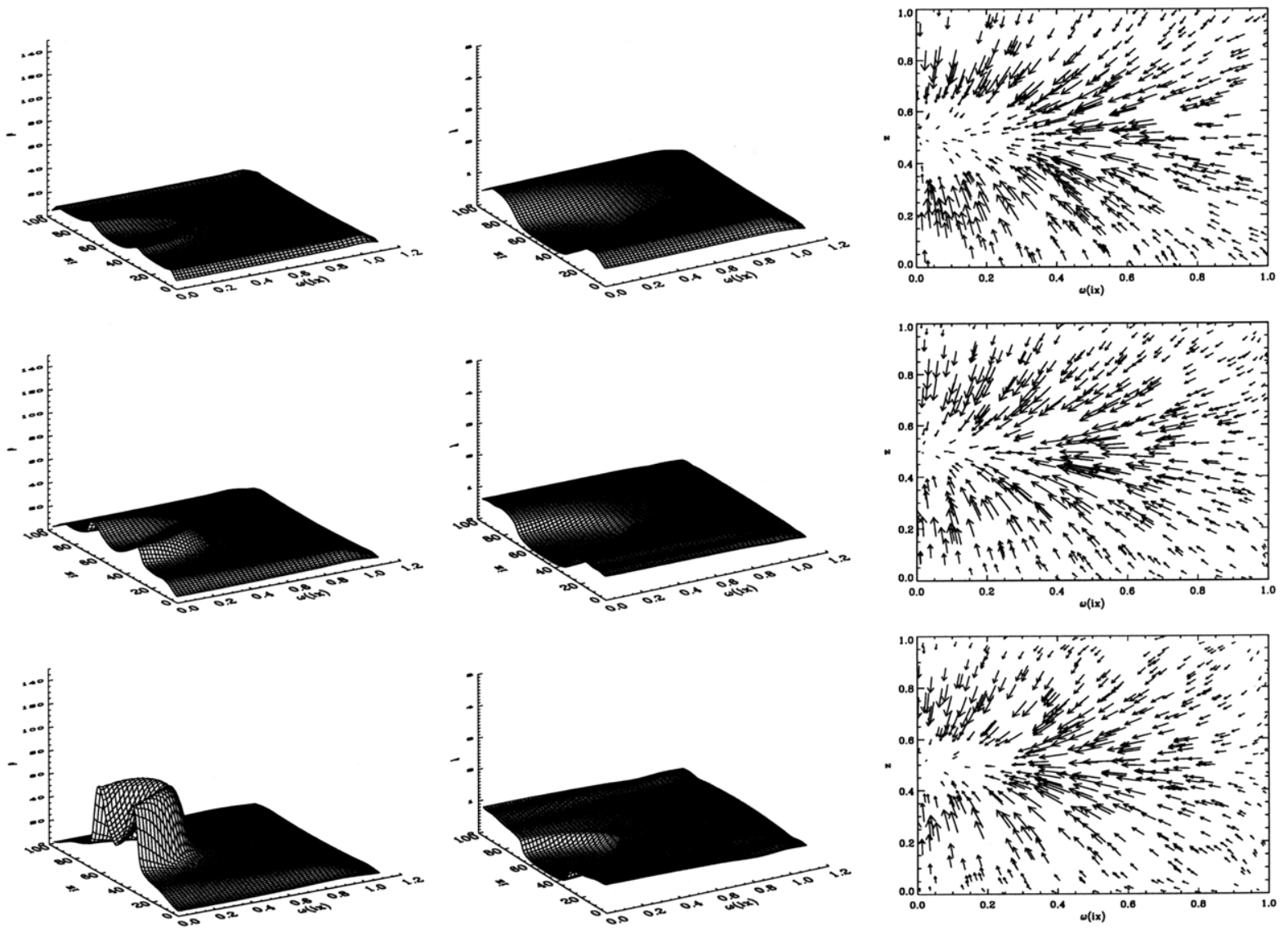

Fig. 15. Time evolution of the density (first column), lambda (second column) and velocity (third column) for the following sequence of times (in units of the initial free-fall time): $3.802 D-03,1.2671,2.723$

for the equilibrium mass density profile in a fixed gravitational potential is $\rho(x)=\rho(0) \mathrm{e}^{-\left(\frac{\mu \mathrm{m}_{\mathrm{H}}}{\mathrm{kT}}\right) \Phi}$ where $\mu$ is the atomic weight, $m_{\mathrm{H}}$ is the mass of a hydrogen atom, $k$ is Boltzmann's constant, $T$ is the temperature, $\Phi$ is the gravitational potential. Because the temperature should be constant throughout the grid at equilibrium, a plot of the numerical values of $\ln (\rho)$ vs. $\Phi$ displays how closely the gas has settled to equilibrium. We plot these quantities for the results from the ECS scheme in Fig. 10. When we fit a line $\ln (\rho)_{i}=a+b \Phi_{i}$ to the numerical results then $a=-2.46484 E-02, b=-15.7485$ and the variance of $a$ is $2.47615 E-05$ while the variance of $b$ is $1.75090 E-03$.

\subsection{On an axisymmetric grid: Gas falling into a fixed external potential}

We simulate the shock heating of gas falling into a fixed, external gravitational potential well in 2D. The hydrodynamic computation is performed on an axisymmetric cylindrical grid which has 50 logarithmically spaced cells in radius, $\varpi$, and 100 evenly spaced cells in the $z$ direction. The gas falls into a gravitational potential well (Fig. 12) which is derived from a spherical density distribution with the following profile: $\rho \sim\left(1+\frac{r^{2}}{r_{\max ^{2}}}\right)^{-2.5}$. The potential is centered on the origin of the axisymmetric cylindrical grid, namely at $\varpi=0$ and at the midplane in $z$. The boundary conditions for the simulation are reflecting. The gas is initially stationary and uniform in density (Fig. 11). As seen in Fig. 11, it is initially higher in temperature at the location of the potential well than in other parts of the grid. In machine units, $G=7, \rho=10$, the radial momentum and vertical momenta densities in each cell are 0, i.e. $P_{\varpi}=0$ and $P_{z}=0$. The internal energy density in each cell is initially set to $\mathcal{E}_{\text {int }}=2.510^{-14}-\mathcal{E}_{\text {grav }}$, where $\mathcal{E}_{\text {grav }}$ is the gravitational energy density in each cell. The axisymmetric cylindrical grid extends from -1.1 to +1.1 in the vertical direction and from 0 to $r_{\max }=+1.1$ in the $\varpi$ direction. We also take $\epsilon$ in the Courant condition to be $.8, \gamma=\frac{5}{3}$, and $\mathcal{C}_{1}=.001$ and $\mathcal{C}_{2}=1$ in the expression for the collision time $\tau$.

Figures 13 and 14 show values of the density, the inverse of the temperature (lambda), and both radial and vertical velocity along two rays through the cylindrical grid. One ray goes through a point $(\varpi=0, \phi=0, z=0)$ and extends in the $\varpi$ direction. We plot various quantities as a function of $\varpi$ along this ray at time $t_{n}$. The second ray 

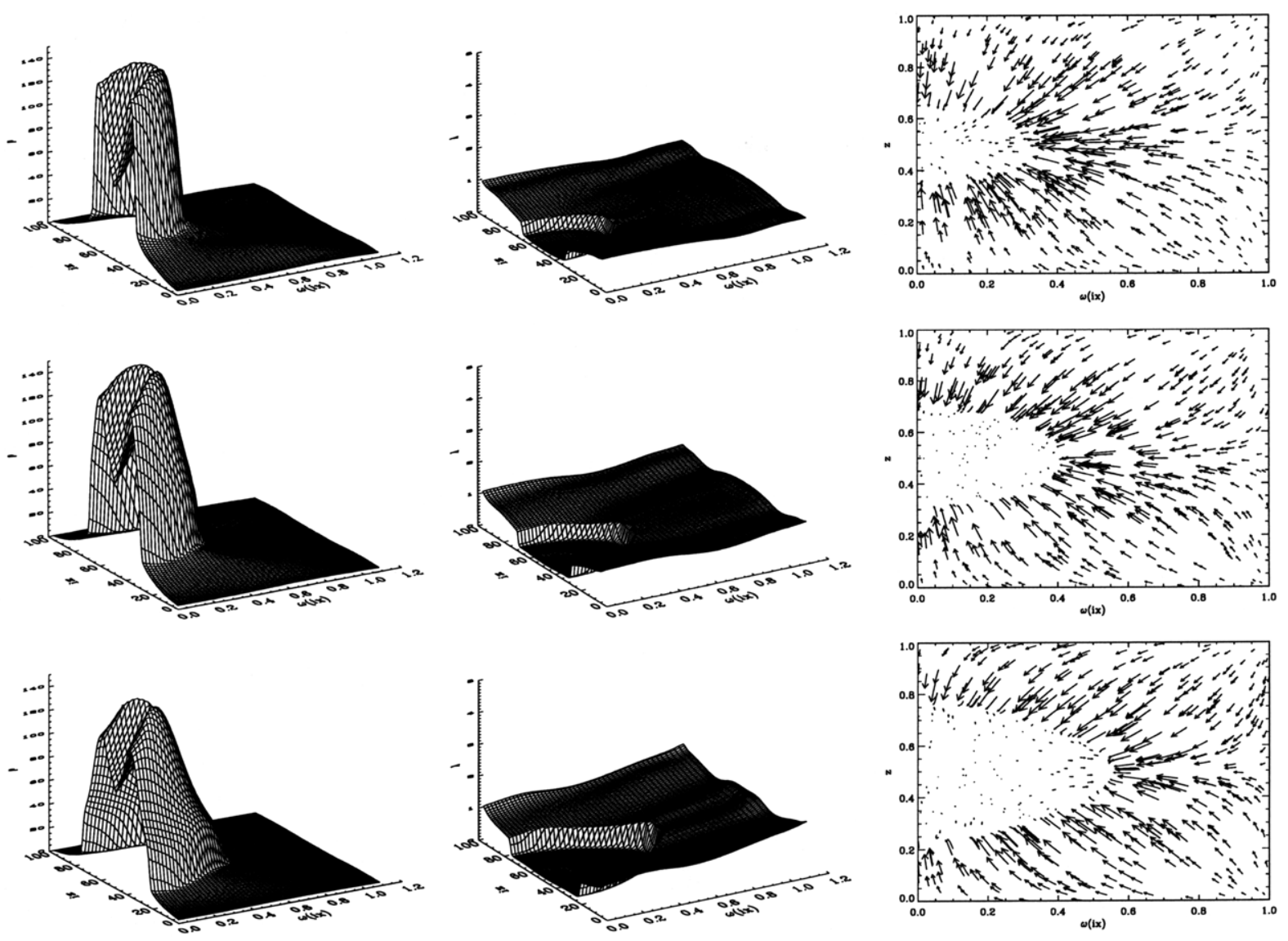

Fig. 16. Continuation of time evolution of the density (first column), lambda (second column) and velocity (third column) for the following sequence of times (in units of the initial free-fall time): $3.607,4.8,5.805$

goes through a point $\left(\varpi=\varpi_{1}, \phi=0, z=-1.1\right)$, extends in the $z$ direction and we plot various quantities as a function of $z$ along this ray at time $t_{n}$. Thus in Figs. 13 and 14 each curve represents results at one instant (specified by $t_{n}$ ) in the course of a run. The curves are approximately evenly spaced in machine time units. The key for the lines styles can be found in the right hand corner of the density plot, (Fig. 13). In the following discussion we will refer primarily to these two Figs. (13 and 14) and to the times for which information is presented in these figures. For a view of the gas evolution in the entire $\varpi-z$ plane, as opposed to merely along these two rays, we will also refer to a time sequence of density and lambda surface plots, as well as to plots of velocity vectors (Figs. 15-17). In the surface plots, values of density and lambda are given on the vertical axis as a function of $\varpi$ and $z$ in the horizontal plane. In the velocity plots, each arrow represents the vector sum of the radial and vertical velocities at a point on the $\varpi-z$ plane. The length of the arrow is proportional to the strength of the velocity field.

From $t_{1}$ through $t_{7}$, the gas density (Fig. 13) rises at the centre of the grid, although the grid centre is not where it reaches its highest values initially (see also Fig. 15). This is because the strong gravitational forces (Fig. 12) keep the gas from falling uniformly into the gravitational potential well. Instead the gas density piles highest along a ring surrounding the grid's centre. The peak of the ring moves inward from $t_{1}$ through $t_{7}$, and the density jump between the tip of the ring and the region outside of it is steepest and reaches a maximum at $t_{8}$ in these figures (see first density plot in Fig. 16). Time $t_{8}$ marks the onset of the outgoing shock into the cold, low-density region outside the ring.

The lambda plots (Fig. 13) show that between $t_{1}$ and $t_{7}$, the region outside the ring becomes progressively colder. This is a consequence of the regions nearer to the ring falling inward more quickly then the regions further from the ring (Fig. 14), leading to an expansion of the gas and a concurrent cooling. By $t_{7}$ the leading edge of the inwardly spreading cold region has steepened into a shock. From $t_{8}$ through $t_{15}$ the heated centre expands sending an outgoing shock which heats the gas as it propagates across the grid. We vividly see both the formation of the shock and its outward movement in the lambda surface plots (Figs. 15-17). In these surface plots, the cooling of the regions further from the ring is also visible.

The formation of the shock is also clearly seen in the velocity profiles (Fig. 14) from $t_{1}$ through $t_{7}$. During this 

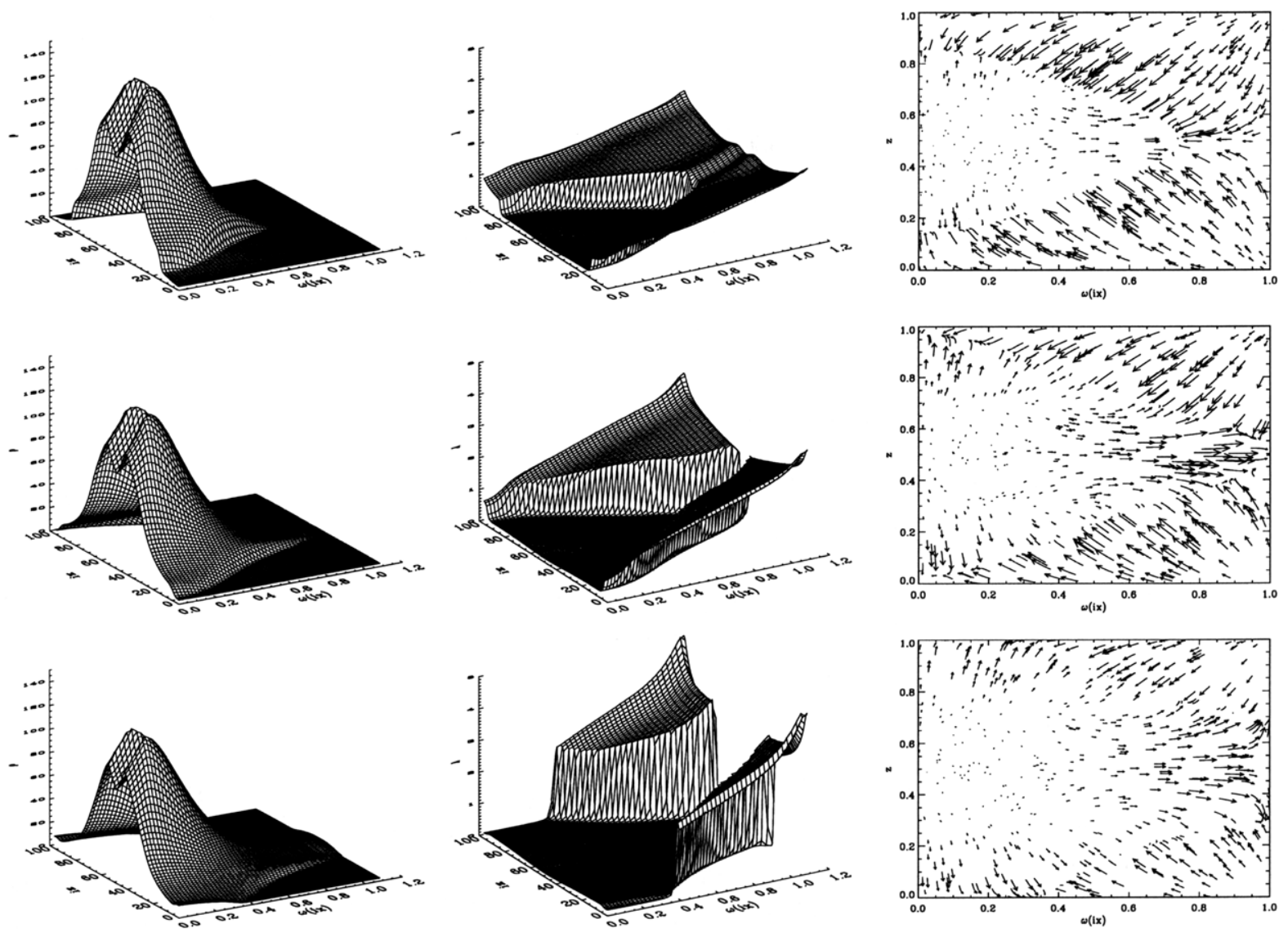

Fig. 17. Continuation of time evolution of the density (first column), lambda (second column) and velocity (third column) for the following sequence of times (in units of the initial free-fall time): 6.898, 8.067, 8.88

time interval, the point of maximum velocity moves inward until at $t_{8}$ the ring material starts moving outward and encounters the material still infalling from the outer part of the grid at a shock front. From $t_{8}$ to $t_{15}$ we see the shock propagating outward. The outwardly moving shock front is delineated clearly in the velocity vector plots (Figs. 15-17).

After 100000 iterations (Fig. 19), the gas is almost in equilibrium: it has a smooth density profile and it is nearly isothermal and stationary. Computing the value of the free-fall time $\left(t_{\mathrm{ff}}=\sqrt{\frac{3 \pi}{32 G \rho}}\right)$ from the initial conditions, $t_{\mathrm{ff}} \approx .06$. Therefore at 100000 iterations (time $=36.3758$ ) the time is 560 in units of the initial free-fall time.

The most significant thing to notice in the results from this computation are the sharp shock fronts. Even though the computation was performed on a relatively coarse grid (50 cells in $\varpi$ and 100 cells in $z$ ), only about two cells are needed to resolve a shock and there is no evidence of under and overshooting. This is shown clearly in Fig. 18, where the inverse of the temperature, $\lambda$, for $t_{9} \rightarrow t_{12}$, is again plotted for the ray which cuts the cylindrical grid along the first cylindrical radius, $\varpi_{1}$ for fixed $\phi=0$. However in Fig. 18, we show the data for each of the four time in- stances in a separate plot, so as to better assess the shock resolution. The circles are the values of $\lambda$ in each cell and the thin lines are curves through these values. It is easy to see that only about two cells are needed to resolve a shock.

\section{Discussion}

The most notable results of the preceeding test runs may be summarized as follows:

1.) For a fixed, external gravitational field both in Cartesian geometry and in axisymmetric cylindrical geometry with a conservative form for the energy equation, the BGK scheme is able to keep a configuration in hydrostatic equilibrium for many sound crossing times. Furthermore with the energy equation in conservative form the BGK scheme settles to the correct physical solution.

2.) The BGK scheme is able to achieve high resolution with rather coarse grids. For example the simulation of gas falling into a fixed external spherically symmetric gravitational potential uses 50 cells in $\varpi$ and 100 cells in $z$ and achieves shocks whose fronts span only 1 or 2 cells. 

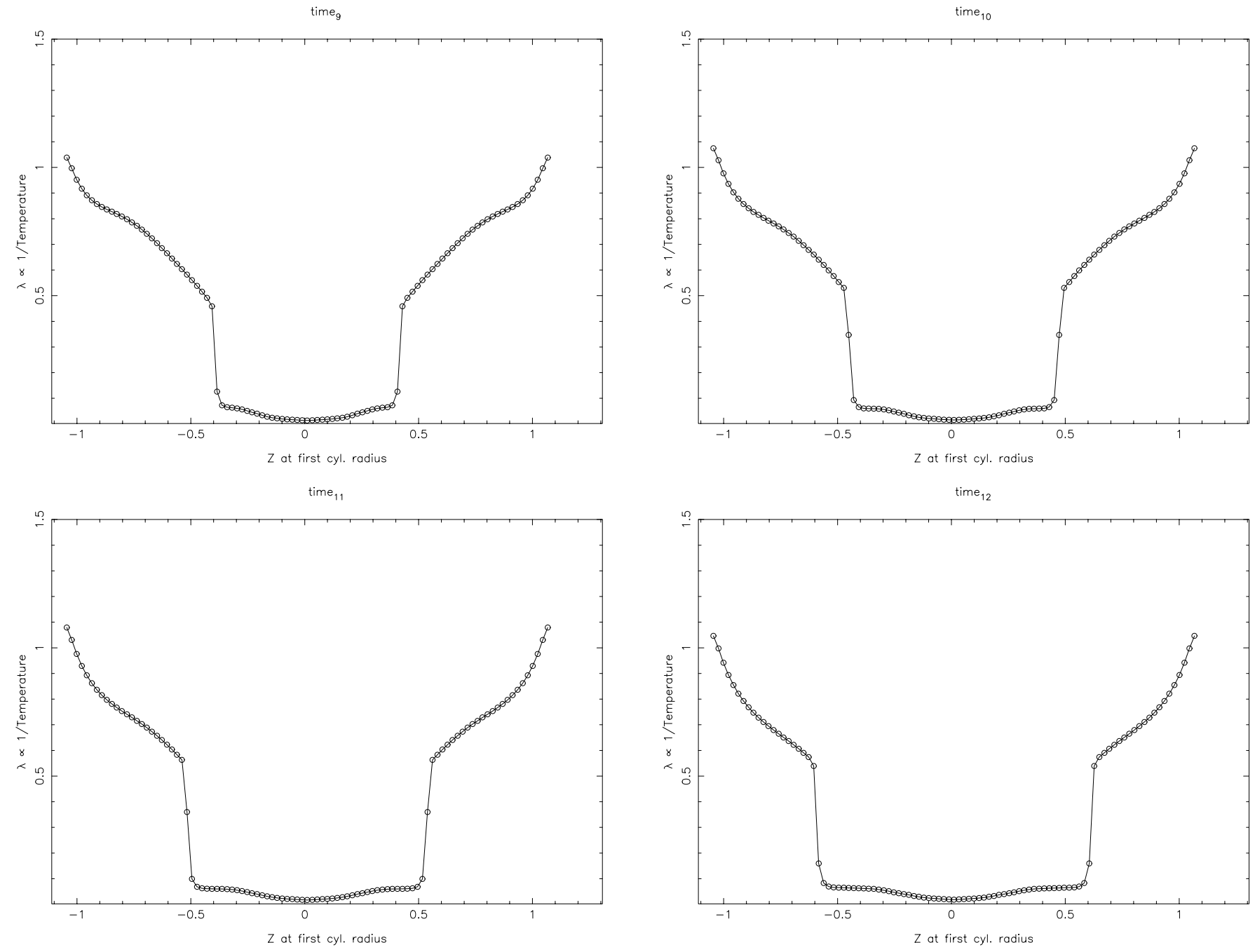

Fig. 18. Lambda plotted once again at different $z$ along $\varpi_{1}$ for $t_{9} \rightarrow t_{12}$. This time the data for each of the four time instances is shown in a separate plot. The circles are the values of $\lambda$ in each cell and the thin lines are curves through these values. Note the sharpness of the shocks and the absence of near-shock oscillations. The shocks have not been sharpened in any way, nor have any oscillations been suppressed; all cells are shown in each plot

We never resort to regridding in the neighborhood of a discontinuity to achieve high resolution. Conventional hydrocodes frequently strive for high resolution through grid refinement techniques. This approach is both expensive and ineffective beyond a certain point. Uniform grid refinement shortens time steps: in 3 dimensions, a decrease by a factor of 2 in the grid spacing of each of the 3 dimensions involves a factor of 16 increase in the number of cycles that the code has to execute to reach the same total time. Furthermore even if one is willing to accept the expense of short timesteps, no matter how fine the grid, a diffusive scheme never achieves the accuracy of a high resolution scheme run on the same sized grid. With that said, local adaptive grid refinement for the BGK scheme (without gravity) has been implemented by Kim \& Jameson (1998) and it gives excellent results for a number of tests involving unsteady supersonic flows.

\section{Conclusion}

We incorporated gravity into the BGK scheme for hydrodynamics and presented results for the case of time-independent gravitational potentials both on a one-dimensional Cartesian grid and on an axisymmetric cylindrical grid. Our results show that the BGK scheme is generalizable to the case of a gas moving in the presence of an external, time-independent gravitational field. When we derive an approximate local solution to the BGK equation, linear in space and time, gravity's curvature of particle trajectories is unimportant. Gravity's effect on the flow enters into the computational method only through gravitational source terms.

We conclude by stating that we believe that no other hydrodynamical scheme which is used in astrophysical computational fluid dynamics invokes as few "fixes" and operates with as much generality as the BGK scheme. 

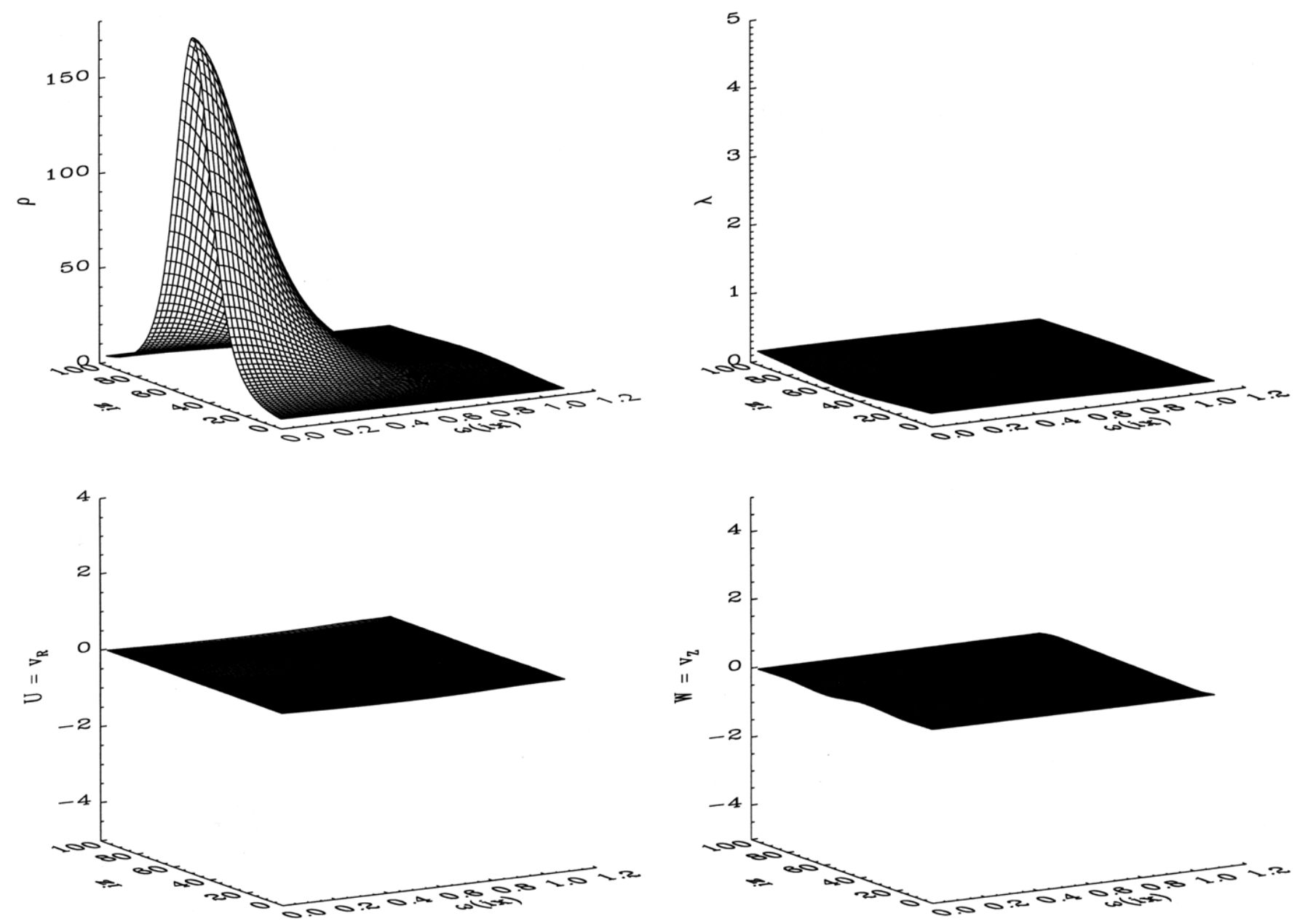

Fig. 19. Density (top left), lambda (top right), radial velocity (bottom left) and vertical velocity (bottom right) after 100000 iterations which is equivalent to a total time in machine units of 36.3758 . This amounts to about 560 free-fall times. Note that lambda is nearly constant and the velocity in both $\varpi$ and $z$ is nearly zero, indicating that the code has (nearly) reached the expected equilibrium state

There are no discontinuity detection algorithms, with subsequent special treatment of special regions. An entropy fix never has to be invoked when following the evolution of rarefaction waves because the BGK scheme satisfies the "entropy condition". Truly multi-dimensional BGK schemes (i.e. non-operator split) are realizable.

We attribute the BGK scheme's performance to its derivation from a model to the collisional Boltzmann equation. A tremendous advantage of a hydrocode designed on the basis of a solution to a model of such a fundamental equation is that the scheme gets physical backing for its incorporation of viscous and heat conductive effects. Velocity moments of a time-dependent local solution to the BGK equation give hydrodynamical fluxes which carry both advective and dissipative terms - the BGK scheme does not decouple them into separate operations. Furthermore the algorithm for solving the BGK equation in the BGK scheme avoids the BGK equation's stiffness which would cause the time step of the scheme to be the collision time of the gas instead of the much larger CFL time. Without such an algorithm to bypass the BGK equation's stiffness, the timestep would be prohibitively small for performing hydrodynamical simulations in real time.

While it may seem complicated to solve for fluid evolution by following a changing distribution function (including the effects of collisions) in phase space, we believe that it is not. In spite of its looks, the BGK scheme is a computationally straightforward explicit scheme, and all parts of the algebra are in explicit closed form for a perfect gas. Even when a matrix is inverted for the connection between the moments and parameters of a Maxwellian, the inverse is explicitly known. Unless readers were told they might not guess from inspection of the code that it originated in gas-dynamic considerations. The code itself is just straightforward algebra. There are no iterative steps in the 
BGK scheme. There is no Riemann solver, either exact or approximate. No numerical sub-steps (e.g. Runge-Kutta steps) are used.

Thus far we have not been concerned with the optimization of the code. Code development has focused on demonstrating that the code correctly simulates physical phenomena. Kim \& Jameson (1998) compared the CPU time required just for the BGK scheme's flux computation to the flux computation in two other high resolution schemes which use flux-splitting methods: characteristic splitting using Roe averaging (Csplit) (Jameson 1996) and CUSP (Convective Upwind Split Pressure) splitting by Jameson (Kim \& Jameson 1995). They find that the BGK scheme is less than twice as slow as the two other schemes. Note that in contrast to the flux computation in the other schemes, the BGK flux computation includes extra arithmetical operations for Navier-Stokes terms. When the other schemes include Navier-Stokes terms, they are not spared this computational expense - it just appears outside of the flux computation.

Acknowledgements. A.S. acknowledges partial support from a NASA Space Grant administered through Cornell University.

\section{References}

Bhatnagar P.L., Gross E.P., Krook M., 1954, Phys. Rev. 94, 511

Cercignani C., 1988, The Boltzmann Equation and its Applications. Springer-Verlag

Chu C.K., 1965, Phys. Fluids. 8, 12

Jameson A., 1996, Int. J. Num. Met. Flu. 20, 743

Kim C., Jameson A., 1995, in 12th AIAA CFD Conference, CP-95-1738

Kim C., Jameson A., 1998, J. Comput. Phys. (in press)

Prendergast K.H., Xu K., 1993, J. Comput. Phys. 109, 53

Roe P.L., 1986, Annu. Rev. Fluid Mech. 18, 337

Shu F.H., 1992, The Physics of Astrophysics, Vol. II, Gas Dynamics. University Science Books

Xu G., 1997, MNRAS 288, 903

Xu K., 1993, Ph.D. thesis, Columbia Univ.

Xu K., Prendergast K.H., 1994, J. Comput. Phys. 114, 9

Xu K., Martinelli L., Jameson A., 1995, J. Comput. Phys. 120, 48

Xu K., Kim L., Martinelli L., Jameson A., 1996, J. Comput. Phys. 7, 213 